\title{
Prestressed stayed beam-columns: Sensitivity to prestressing levels, pre-cambering and imperfections
}

\author{
Kaidong Wu, M. Ahmer Wadee, Leroy Gardner \\ Department of Civil and Environmental Engineering, Imperial College London, \\ South Kensington Campus, London SW7 2AZ, UK
}

\begin{abstract}
The behaviour and structural performance of imperfect beam-columns with crossarms and externally prestressed cable stays are studied numerically, where the combination of bending and compression is assumed to be derived from the system self-weight acting orthogonally to the applied axial load. Both doubly-symmetric and mono-symmetric systems are studied. Sensitivity of the structural response to varying prestressing levels, pre-cambering and initial imperfections is investigated. Different initial imperfection levels and combinations are considered to facilitate the exploration of interactive buckling. The optimum prestressing force in terms of ultimate resistance and two structural efficiency indicators is also studied. It is found that relatively small crossarm lengths, stay diameters and crossarm length ratios should be avoided. Moreover, mono-symmetric cases are more sensitive to the level of pre-cambering than their doubly-symmetric counterparts. Considering both load-carrying capacity and structural efficiency, doubly-symmetric cases perform best with zero pre-cambering, but mono-symmetric cases perform best with upward precambering. As for the true optimum prestressing levels, these are recommended to be significantly above the linearly obtained optimum to maximize the structural efficiency.
\end{abstract}

Keywords: Prestressed stayed beam-columns; Imperfections; Pre-cambering; Finite element modelling; Interactive buckling; Steel structures

\section{Introduction}

Slender structural components supported with prestressed cable-stays in conjunction with periodically-spaced crossarms distributed longitudinally, offer an attractive, innovative and pragmatic solution to reducing structural self-weight while maintaining buckling strength. The use of the crossarm-stay system with pretensioning within the cable stays effectively provides intermediate supports that act to reduce the effective buckling wavelength of the main member which, in turn, results in a higher load-carrying capacity.

\footnotetext{
*Corresponding author

Email addresses: k.wu17@imperial.ac.uk (Kaidong Wu), a.wadee@imperial.ac.uk (M. Ahmer Wadee), leroy.gardner@imperial.ac.uk (Leroy Gardner)
} 
Research on this type of structural system can be found initially from the 1960s [1, 2] with specific work on determining the buckling response analytically $[3,4]$ and the important relationship between the levels of pretensioning and the critical buckling load [5]. The work in [3] was verified in [6,7], while imperfection sensitivity was investigated in [8]. Experiments and numerical studies were conducted to confirm the buckling behaviour $[9,10,11,12]$, and the stability of multiple-crossarm prestressed stayed steel columns has also been investigated $[13,14]$. Subsequently, the relationship regarding the levels of prestress and the nonlinear post-buckling behaviour of stayed struts has been determined numerically [15], theoretically for distinct post-buckling behaviour [16, 17], and where interactive buckling occurs $[18,19,20,21]$. Design guidance for struts with one intermediate crossarm has been presented in $[22,23]$. In addition, prestressed steel elements under tension and compression were studied in $[24,25]$, while, more recently, the stability and design of prestressed cold-formed steel members have also been investigated [26, 27, 28, 29].

Most of the aforementioned research work has been conducted for vertical members where the directions of the self-weight and the axial load have been collinear. As discussed in [30], there is a potential demand for very long struts to be used in the horizontal plane, for example to support excavation works in the construction of large scale foundations. Existing so-called 'super struts' are currently used; constructed from heavy tubular steel cross-sections, these struts need to withstand earth pressures axially in conjunction with their considerable self-weight under bending. An iconic structure that has already employed a system of stayed columns and beam-columns is 'La Grande Arche' in Paris [31]. The internal structure includes a suspended membrane roof, part of which is supported by a system of prestressed cable-stayed columns that are braced every $21 \mathrm{~m}$ by stayed beamcolumns in the horizontal plane, the details of which are shown in Figure 1.

In [30], a prestressed stayed beam-column was studied as a viable alternative to super struts - see Figure 2. The present work continues the study on stayed beam-columns placed in the horizontal plane where self-weight becomes more important. The self-weight introduces a directionality similar to that found in certain cable-stayed bridges, where stays are regularly used to reduce the self-weight deflection of the deck, thereby increasing structural capacity [32]. It has already been demonstrated in [30] that proportioning the prestress in the upper and lower stays such that the crossarms have a net zero axial force at mid-span results in the effect of the self-weight being counteracted and approximately maximizes performance. Moreover, it was also determined that two of the three zones of mechanical behaviour, which were initially discovered in [5] are each sub-divided into two further sub-zones of behaviour owing to the effect of the self-weight. However, the investigation presented in [33] found that the theoretical compressive strength enhancements under certain configurations can only be obtained at the expense of triggering a sequence of destabilizing bifurcations. This can give rise to severely unstable interactive post-buckling behaviour including the so-called 'mode jumping' phenomenon [34]. Following the recent study on the zonal behaviour, the linear optimum prestressing forces, determined in [30], are used as the bases for the present study. Moreover, from the mode interaction study in [33], different imperfection combinations are adopted to take account of the weakest case leading to interactive buckling. 


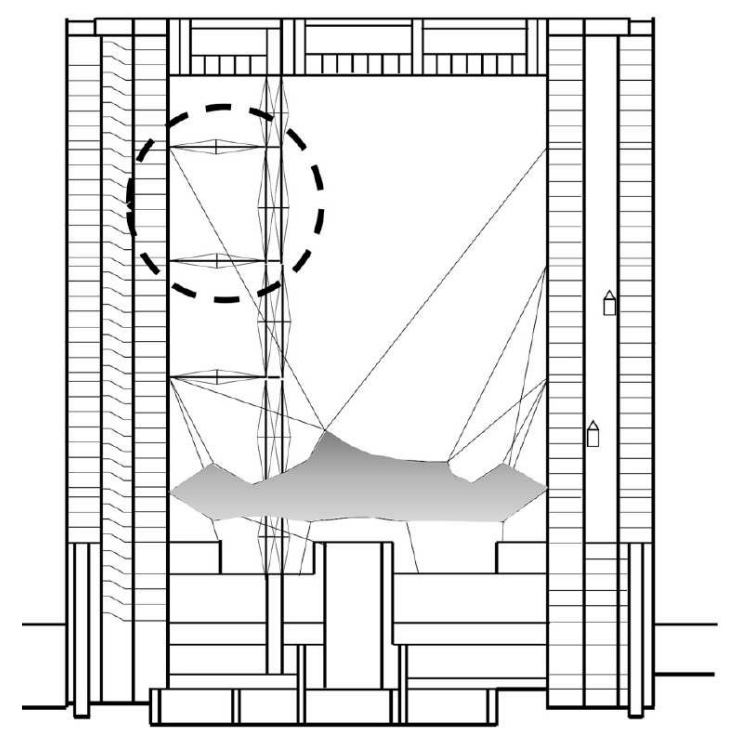

(a)

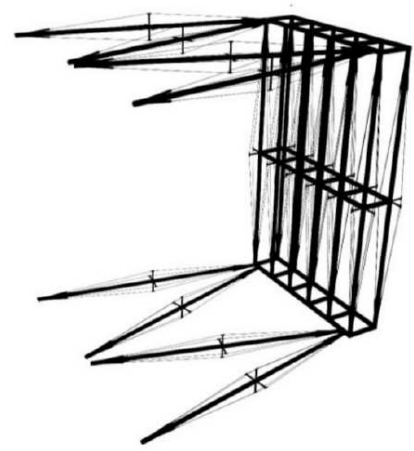

(b)

Figure 1: Application of prestressed stayed columns and beam-columns within La Grande Arche in Paris: (a) structural elevation; (b) detail from the circled region in (a) showing stayed elements in the vertical and horizontal plane. Schematics have been taken from [31].
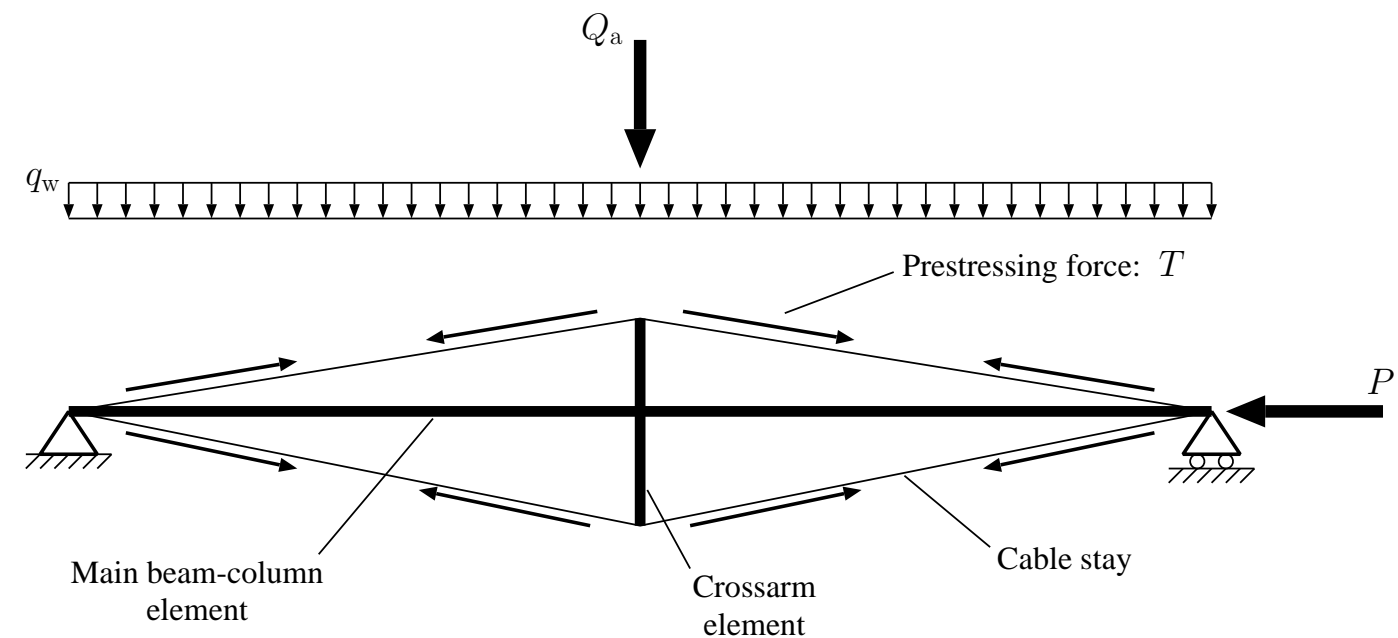

Figure 2: Prestressed stayed beam-column, under an axial load $P$ with self-weight lateral loads $Q_{\mathrm{a}}$ from the crossarm and $q_{\mathrm{w}}$ from the beam-column element; cable stays are under an initial prestressing force $T$ that place both the beam-column element and crossarms under axial compression before any external loading is introduced. 
Presently, numerical models are employed to study the structural response of imperfect stayed beam-columns. Both doubly-symmetric and mono-symmetric systems are examined. Although previous studies have investigated the stability of this system, their focus was only on stayed beam-columns with 'perfect' geometries under a combination of axial compression and self-weight. The current study investigates the structural performance of imperfect stayed beam-columns under axial compression in conjunction with their selfweight numerically. Different imperfection combinations are considered for interactive buckling, and the effects on the structural behaviour from varying prestressing and precambering levels are investigated. The optimum prestressing force for ultimate strength, referred to as the true optimum prestressing force, is determined based on the ultimate load achieved and two bespoke structural efficiency indicators. The structural performance is found to be strongly linked to the level of pre-cambering.

\section{General definitions and modelling}

The numbering of the stays and the geometric parameters of the currently considered prestressed stayed beam-column are shown in Figure 3. The upper and lower crossarm

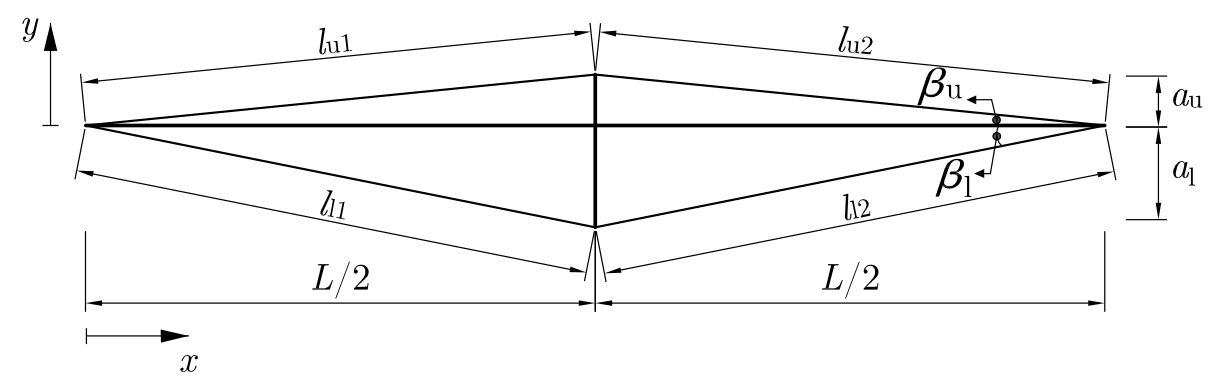

Figure 3: Stay numbering and geometric parameters of the prestressed stayed beam-column: $L$ is the total length of the main beam-column element; $a_{\mathrm{u}}$ and $a_{\mathrm{l}}$ are the lengths of the upper and lower crossarms respectively; $\beta_{\mathrm{u}}$ and $\beta_{\mathrm{l}}$ are the angles between the main member with the upper and lower stays respectively; $l_{\mathrm{un}}$ and $l_{\mathrm{ln}}$ represent the lengths of the upper and lower stays respectively, where $\mathrm{n}=\{1,2\}$. Currently, $l_{\mathrm{u}}=l_{\mathrm{u} 1}=l_{\mathrm{u} 2}$ and $l_{1}=l_{11}=l_{12}$.

ratios are defined respectively as follows:

$$
\alpha_{\mathrm{u}}=\frac{a_{\mathrm{u}}}{L}, \quad \alpha_{1}=\frac{a_{\mathrm{l}}}{L},
$$

and the crossarm length ratio $\alpha$ is introduced thus:

$$
\alpha=\frac{\alpha_{\mathrm{u}}}{\alpha_{\mathrm{l}}} .
$$

Based on the geometry shown in Figure 3, the length of each stay can be expressed thus:

$$
\begin{aligned}
& l_{\mathrm{u}}=l_{\mathrm{u} 1}=l_{\mathrm{u} 2}=L \sqrt{\alpha_{\mathrm{u}}^{2}+1 / 4}, \\
& l_{\mathrm{l}}=l_{\mathrm{l} 1}=l_{\mathrm{l} 2}=L \sqrt{\alpha_{1}^{2}+1 / 4} .
\end{aligned}
$$


As in previous work $[16,12,20]$, the connections between the crossarms and the main column element are assumed to be rigid, whereas the connections between the stay cables and both the main column element and the crossarms are assumed to be pinned.

Presently, the upper and lower crossarm lengths ( $a_{\mathrm{u}}$ and $a_{1}$ respectively) and stay diameter $\phi_{\mathrm{s}}$ are varied, while the material properties and structural dimensions of the prestressed stayed beam-columns are fixed to the values listed in Table 1. In terms of

Table 1: Material properties and structural dimensions of the prestressed stayed beam-columns in the current numerical study.

\begin{tabular}{ll}
\hline Length of main member & $L=3050 \mathrm{~mm}$ \\
Main member Young's moduli & $E_{\mathrm{c}}=201 \mathrm{kN} / \mathrm{mm}^{2}$ \\
Crossarm Young's moduli & $E_{\mathrm{a}}=201 \mathrm{kN} / \mathrm{mm}^{2}$ \\
Stay Young's moduli & $E_{\mathrm{s}}=202 \mathrm{kN} / \mathrm{mm}^{2}$ \\
Outside diameter of main member & $\phi_{\mathrm{co}}=38.1 \mathrm{~mm}$ \\
Inside diameter of main member & $\phi_{\mathrm{ci}}=25.4 \mathrm{~mm}$ \\
Outside diameter of crossarm & $\phi_{\mathrm{ao}}=38.1 \mathrm{~mm}$ \\
Inside diameter of crossarm & $\phi_{\mathrm{ai}}=25.4 \mathrm{~mm}$ \\
Material density (steel) & $\rho=7850 \mathrm{~kg} / \mathrm{m}^{3}$ \\
Gravitational acceleration & $g=9.81 \mathrm{~m} / \mathrm{s}^{2}$ \\
Yield stress of main member & $f_{\mathrm{y}}=340 \mathrm{~N} / \mathrm{mm}^{2}$ \\
\hline
\end{tabular}

doubly-symmetric (i.e. $\alpha=1$ ) cases, 12 configurations, as defined in [18], listed as F1-F6 and A1-A6 are studied (see Table 2). In addition, 9 further mono-symmetric (i.e. $\alpha<1$ ) model configurations, listed as F3.1-F3.9 are investigated (see Table 3). The normalized

Table 2: Parameters of studied doubly-symmetric $(\alpha=1.0)$ cases, where $\alpha_{1}=0.1$ in cases F1-F6 and $\phi_{\mathrm{s}} /\left(2 r_{\mathrm{c}}\right)=0.21$ in cases $\mathrm{A} 1-\mathrm{A} 6 ; r_{\mathrm{c}}$ is the main column element radius of gyration.

\begin{tabular}{ccccccc}
\hline Case & F1 & F2 & F3 & F4 & F5 & F6 \\
\hline$\phi_{\mathrm{s}}(\mathrm{mm})$ & 1.6 & 3.2 & 4.8 & 6.4 & 8.0 & 10.0 \\
$\phi_{\mathrm{s}} /\left(2 r_{\mathrm{c}}\right)$ & 0.07 & 0.14 & 0.21 & 0.28 & 0.35 & 0.44 \\
\hline Case & $\mathrm{A} 1$ & $\mathrm{~A} 2$ & $\mathrm{~A} 3$ & $\mathrm{~A} 4$ & $\mathrm{~A} 5$ & $\mathrm{~A} 6$ \\
\hline$\alpha_{1}$ & 0.025 & 0.05 & 0.075 & 0.100 & 0.125 & 0.150 \\
\hline
\end{tabular}

Table 3: Parameters of studied mono-symmetric $(\alpha<1.0)$ cases, where $\alpha_{1}=0.1$ and $\phi_{\mathrm{s}} /\left(2 r_{\mathrm{c}}\right)=0.21$.

\begin{tabular}{cccccccccc}
\hline Case & F3.1 & F3.2 & F3.3 & F3.4 & F3.5 & F3.6 & F3.7 & F3.8 & F3.9 \\
\hline$\alpha$ & 0.1 & 0.2 & 0.3 & 0.4 & 0.5 & 0.6 & 0.7 & 0.8 & 0.9 \\
\hline
\end{tabular}

slenderness of the main member element $\bar{\lambda}$ is defined by the well known expression [35]:

$$
\bar{\lambda}=\sqrt{P_{\mathrm{y}} / P_{\mathrm{E}}}
$$


where $P_{\mathrm{y}}$ is the squash load and $P_{\mathrm{E}}$ is the Euler buckling load of the main member element, thus:

$$
P_{\mathrm{y}}=A_{\mathrm{c}} f_{\mathrm{y}}, \quad P_{\mathrm{E}}=\frac{\pi^{2} E_{\mathrm{c}} I_{\mathrm{c}}}{L^{2}},
$$

where $I_{\mathrm{c}}$ and $A_{\mathrm{c}}$ are the main member second moment of area about the axis of bending and the cross-sectional area of the main member respectively. For the main member within the current study, $P_{\mathrm{y}}=214 \mathrm{kN}, P_{\mathrm{E}}=17.7 \mathrm{kN}$ and $\bar{\lambda}=3.477$. Note also that the main member radius of gyration, $r_{\mathrm{c}}=\sqrt{I_{\mathrm{c}} / A_{\mathrm{c}}}$, is $11.45 \mathrm{~mm}$.

\subsection{Finite element (FE) modelling}

Numerical FE simulations, developed following the approach described in [36, 37], are used to study the beam-column system currently. Following previous numerical studies of prestressed stayed columns using the commercial software ABAQUS [18, 12, 20], the Euler-Bernoulli beam element B23 is adopted to model the main horizontal member as well as the crossarms. Since all the stays are pinned to the ends of the main member and crossarms, these are modelled with the truss element T2D2 in conjunction with the "no compression' option. Taking guidance from the previous study [20], the main member and crossarms are discretized using the aforementioned B23 beam element with a length of $50 \mathrm{~mm}$, while each stay component is taken as an individual T2D2 truss element. Buckling analysis is conducted to calculate the linear eigenvalue and eigenmode profile, and then Riks analysis [38] is conducted to obtain the nonlinear post-buckling behaviour.

In a similar fashion to the numerical study of interactive buckling within imperfect stayed columns [18], an initial imperfection shape $W_{0}$ for the main beam-column element is introduced, thus:

$$
W_{0}=\delta L\left[\mu_{1} \sin \frac{\pi x}{L}+\mu_{2} \sin \frac{2 \pi x}{L}\right] .
$$

In this expression, $\delta$ is the imperfection amplitude ratio with respect to the column length $L$, whereas $\mu_{1}$ and $\mu_{2}$ are scale factors introduced to represent the proportions of the initial imperfection shape of the stayed beam-column that are affine to buckling modes 1 (i.e. a single half sine wave) and 2 (i.e. a full sine wave) of regular (unstayed) prismatic columns respectively. The relationship between $\mu_{1}$ and $\mu_{2}$ is given by:

$$
\mu_{1}^{2}+4 \mu_{2}^{2}=1
$$

which was determined from the basis that different initial imperfection shapes should have an equal initial end-shortening displacement such that a fair comparison can be made between imperfection shapes of different wavelengths [39]. This relationship is used to introduce an initial imperfection to the stayed beam-column geometry to trigger either a pure buckling mode or an interactive nonlinear buckling mode within the FE modelling. The considered imperfection combination cases are listed in Table 4. 
Loading step 1: Applying crossarm and main member self-weight $Q_{\mathrm{a}}$ and $q_{\mathrm{w}}$

$q_{\mathrm{w}}, Q_{\mathrm{a}}$

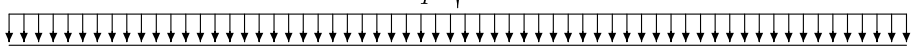

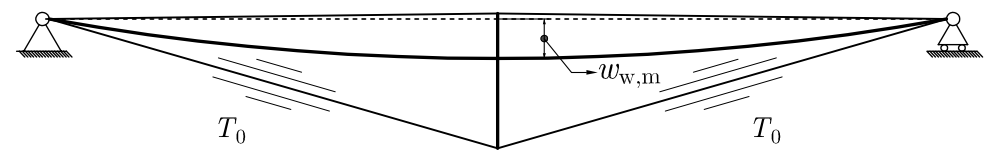

Loading step 2: Prestressing to counteract self-weight;

increasing tension in the lower stays to $T_{11, \mathrm{w}}, T_{12, \mathrm{w}}$ such that $w_{\mathrm{w}, \mathrm{m}}=0$

$q_{\mathrm{w}}, Q_{\mathrm{a}}$

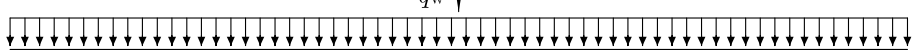

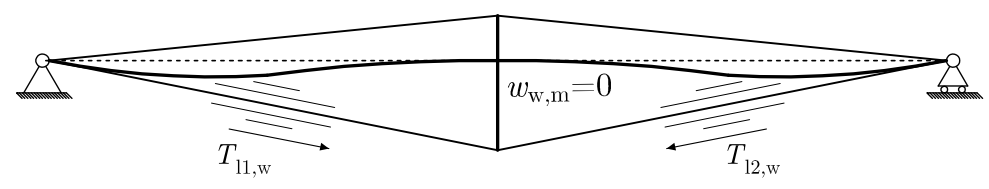

Loading step 3: Prestressing to improve buckling performance; increasing tension in all stays simultaneously to $T_{\mathrm{u} 1, \mathrm{i}}, T_{\mathrm{u} 2 \mathrm{i},}, T_{11, \mathrm{i}}, T_{12, \mathrm{i}}$ such that the axial forces within the crossarms are equal (to leading order)

$q_{\mathrm{w}}, Q_{\mathrm{a}}$

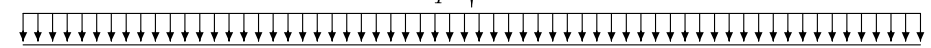

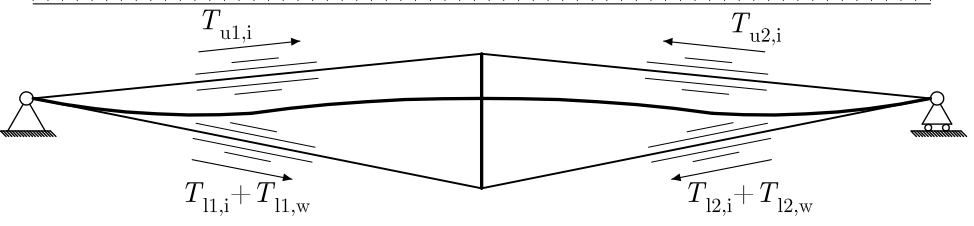

Loading step 4: Applying compressive axial load $P$ until system buckles

$q_{\mathrm{w}}, Q_{\mathrm{a}}$
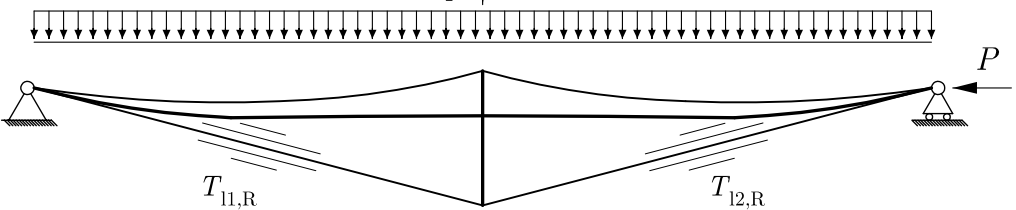

Figure 4: Loading steps for the prestressed stayed beam-column. In steps 1-3, all stays are assumed to remain straight; $T_{0}$ is the force within the lower stays in step $1 ; w_{\mathrm{w}, \mathrm{m}}$ is the mid-span vertical displacement after applying self-weight and $T_{1, \mathrm{w}}=T_{11, \mathrm{w}}=T_{12, \mathrm{w}}$ are the prestressing forces within the lower stays to counteract the mid-span self-weight deflection; $T_{\mathrm{u}, \mathrm{i}}=T_{\mathrm{u} 1, \mathrm{i}}=T_{\mathrm{u} 2, \mathrm{i}}$ and $T_{1, \mathrm{i}}=T_{11, \mathrm{i}}=T_{12, \mathrm{i}}$ are the prestressing forces applied within the upper and lower stays respectively to increase the structural capacity, and $T_{\mathrm{u}, \mathrm{i}} \sin \beta_{\mathrm{u}}=T_{1, \mathrm{i}} \sin \beta_{1}[30] ; T_{1, \mathrm{R}}=T_{11, \mathrm{R}}=T_{12, \mathrm{R}}$ are the residual tensions within the lower stays when the system buckles. 
Table 4: Imperfection combination cases: cases 1 and 5 represent pure eigenmode imperfections for modes 1 and 2 respectively; cases $2-4$ represent those that are designed to trigger interactive buckling responses.

\begin{tabular}{cccccc}
\hline Case & 1 & 2 & 3 & 4 & 5 \\
\hline$\mu_{1}$ & 1.000 & 0.750 & 0.500 & 0.250 & 0.000 \\
$\mu_{2}$ & 0.000 & 0.331 & 0.433 & 0.484 & 0.500 \\
\hline
\end{tabular}

\subsection{Loading steps and assumptions}

Similar to the studies on the stayed beam-column presented in [30, 33], the loading steps illustrated in Figure 4 are adopted. This procedure takes the amplification of the bending displacement from self-weight into account. If the self-weight were not considered, steps 1-2 could be omitted, and within steps 3-4, the following quantities would have zero magnitudes: $q_{\mathrm{w}}, Q_{\mathrm{a}}, T_{11, \mathrm{w}}$ and $T_{12, \mathrm{w}}$. Within step 3 , the relationship between the prestressing forces within the upper and lower stays, defined as $T_{\mathrm{u}, \mathrm{i}}$ and $T_{1, \mathrm{i}}$ respectively, is given by:

$$
T_{\mathrm{u}, \mathrm{i}} \sin \beta_{\mathrm{u}}=T_{\mathrm{l}, \mathrm{i}} \sin \beta_{\mathrm{l}} .
$$

The aim of satisfying this relationship is to achieve zero pre-cambering at mid-span after step 3; satisfying Eq. (8) was shown in [30] to maximize the structural performance approximately. However, it is shown later that zero pre-cambering is not achieved by satisfying Eq. (8) owing to the end-shortening induced by prestressing the stays; this issue is investigated in Sections 3 and 4, where the combined effect of imperfections and pre-cambering is studied.

For modelling purposes, the following assumptions are made:

1. The effects of geometrical nonlinearity from combining bending and axial compression are included.

2. All materials are linearly elastic since the geometries are very slender and hence the strains, are in general, substantially below the yield strain.

3. The self-weight of all the stays is neglected since the unit-length self-weight of the stays is less than $10 \%$ of that from the main member in the vast majority of practical cases.

\subsection{Benchmark prestressing force}

The zonal behaviour and expressions for the benchmark prestressing forces for prestressed stayed beam-columns under self-weight and axial compression were identified in [30] and a summary of the results follows. Based on the axial force definitions within loading step 4 in [30], $T_{\mathrm{u}, \mathrm{i}}$ and $T_{\mathrm{u}, \mathrm{f}}$ are the initially applied prestressing force and the final force at buckling respectively within the upper stays; $T_{1, \mathrm{i}}$ and $T_{1, \mathrm{f}}$ are the initially applied prestressing forces in addition to $T_{1, \mathrm{w}}$ and the final force at buckling respectively within the lower stays. These prestressing forces are applied to the stays to increase the load-carrying capacity of the main member.

In terms of the specific case where $\alpha=1.0$ (i.e. equal length crossarms), the three zones of behaviour were identified in $[5,16]$ and the characteristic graph is presented in 
Figure 5(a). In terms of the cases where $\alpha<1.0$, there exist sub-zones within both Zones 2 and 3 [30]. The zones of behaviour where $\alpha<1.0$ are illustrated in Figure 5(b). Details of the responses within each zone can be described as follows:

Zone 1 - when $T_{1, \mathrm{i}}=\left[0, T_{\min 21}\right]$, corresponding to $T_{\mathrm{u}, \mathrm{i}}=\left[0, T_{\min 2}\right]$, on applying $P$, the prestressing forces would disappear owing to the axial end-shortening displacement before Euler buckling occurs, and so the response replicates that of an unstayed beam-column. For the case where $\alpha=1.0, T_{1, \mathrm{i}}=T_{\mathrm{u}, \mathrm{i}}=\left[0, T_{\min }\right]$.

Zone 2 - when $T_{1, \mathrm{i}}=\left[T_{\min 21}, T_{\mathrm{opt} 21}\right]$, corresponding to $T_{\mathrm{u}, \mathrm{i}}=\left[T_{\min 2}, T_{\mathrm{opt} 2}\right]$, on applying $P$, the prestressing forces within all the stays would disappear when the system buckles, generally at a higher load compared to Zone 1 . However, there are two different subzones within Zone 2, i.e. 'Zone 2-1' and 'Zone 2-2' that are characterized by whether or not a residual force within the lower stays exists when the external load $P=P_{\mathrm{E}}$, thus:

Zone 2-1 - when $T_{1, \mathrm{i}}=\left[T_{\min 21}, T_{\min 1}\right]$, corresponding to $T_{\mathrm{u}, \mathrm{i}}=\left[T_{\min 2}, T_{\min 12}\right]$, and $P=P_{\mathrm{E}}$, there is a residual force within the upper stays $\left(i . e . T_{\mathrm{u}, \mathrm{f}}>0\right.$ ) while $T_{\mathrm{l}, \mathrm{f}}=0$ within the lower stays. The restraining effect from the residual force within only the upper stays increases $P^{\mathrm{C}}$, where $P^{\mathrm{C}}=\left[P_{\mathrm{E}}, P_{\max , 2-1}^{\mathrm{C}}\right]$, with $P_{\max , 2-1}^{\mathrm{C}}$ being the maximum critical buckling load in this sub-zone.

Zone 2-2 - when $T_{1, \mathrm{i}}=\left[T_{\min 1}, T_{\mathrm{opt} 21}\right]$, corresponding to $T_{\mathrm{u}, \mathrm{i}}=\left[T_{\min 12}, T_{\mathrm{opt} 2}\right]$, and $P=P_{\mathrm{E}}$, there is a residual force within both the upper and lower stays, i.e. $T_{\mathrm{u}, \mathrm{f}}>0$ and $T_{\mathrm{l}, \mathrm{f}}>0$. The restraining effect from the residual forces within both the upper and lower stays increases $P^{\mathrm{C}}$ further, and $P^{\mathrm{C}}=\left[P_{\max , 2-1}^{\mathrm{C}}, P_{\max , 2-2}^{\mathrm{C}}\right]$, where $P_{\max , 2-2}^{\mathrm{C}}$ is the maximum critical buckling load in this sub-zone (referred to previously as $P_{\max , 1}^{\mathrm{C}}$ in [30]).

For the case where $\alpha=1.0$, there is no such subdivision of the zone and $T_{1, \mathrm{i}}=T_{\mathrm{u}, \mathrm{i}}=$ $\left[T_{\min }, T_{\text {opt }}\right]$.

Zone 3 - when $T_{1, \mathrm{i}}>T_{\mathrm{opt} 21}$, corresponding to $T_{\mathrm{u}, \mathrm{i}}>T_{\mathrm{opt} 2}$, on applying $P$, the beamcolumn buckles while residual forces exist within all the stays. However, in a similar fashion to Zone 2, there are two different sub-zones within Zone 3, i.e. 'Zone 3-1' and 'Zone 3-2' that are characterized by whether or not a residual force within the lower stays exists at the point of buckling, thus:

Zone 3-1 - when $T_{1, \mathrm{i}}=\left[T_{\mathrm{opt} 21}, T_{\mathrm{opt} 1}\right]$, corresponding to $T_{\mathrm{u}, \mathrm{i}}=\left[T_{\mathrm{opt} 2}, T_{\mathrm{opt12}}\right]$, on applying $P, T_{1, \mathrm{i}}$ would reduce to zero, but there is a residual force within the upper stays $\left(T_{\mathrm{u}, \mathrm{f}}>0\right)$ when the system buckles; the latter decreases $P^{\mathrm{C}}$, and $P^{\mathrm{C}}=\left[P_{\max , 3-1}^{\mathrm{C}}, P_{\max , 2-2}^{\mathrm{C}}\right]$, where $P_{\max , 3-1}^{\mathrm{C}}$ marks the minimum critical buckling load in this sub-zone (referred to previously as $P_{\max , 2}^{\mathrm{C}}$ in [30]).

Zone 3-2 - when $T_{1, \mathrm{i}}>T_{\mathrm{opt} 1}$, corresponding to $T_{\mathrm{u}, \mathrm{i}}>T_{\mathrm{opt} 12}$, on applying $P$, the force within both the lower and upper stays both have residual forces when the system buckles, i.e. $T_{\mathrm{l}, \mathrm{f}}>0$ and $T_{\mathrm{u}, \mathrm{f}}>0$, these decrease $P^{\mathrm{C}}$, and $P^{\mathrm{C}}<P_{\max , 3-1}^{\mathrm{C}}$. 

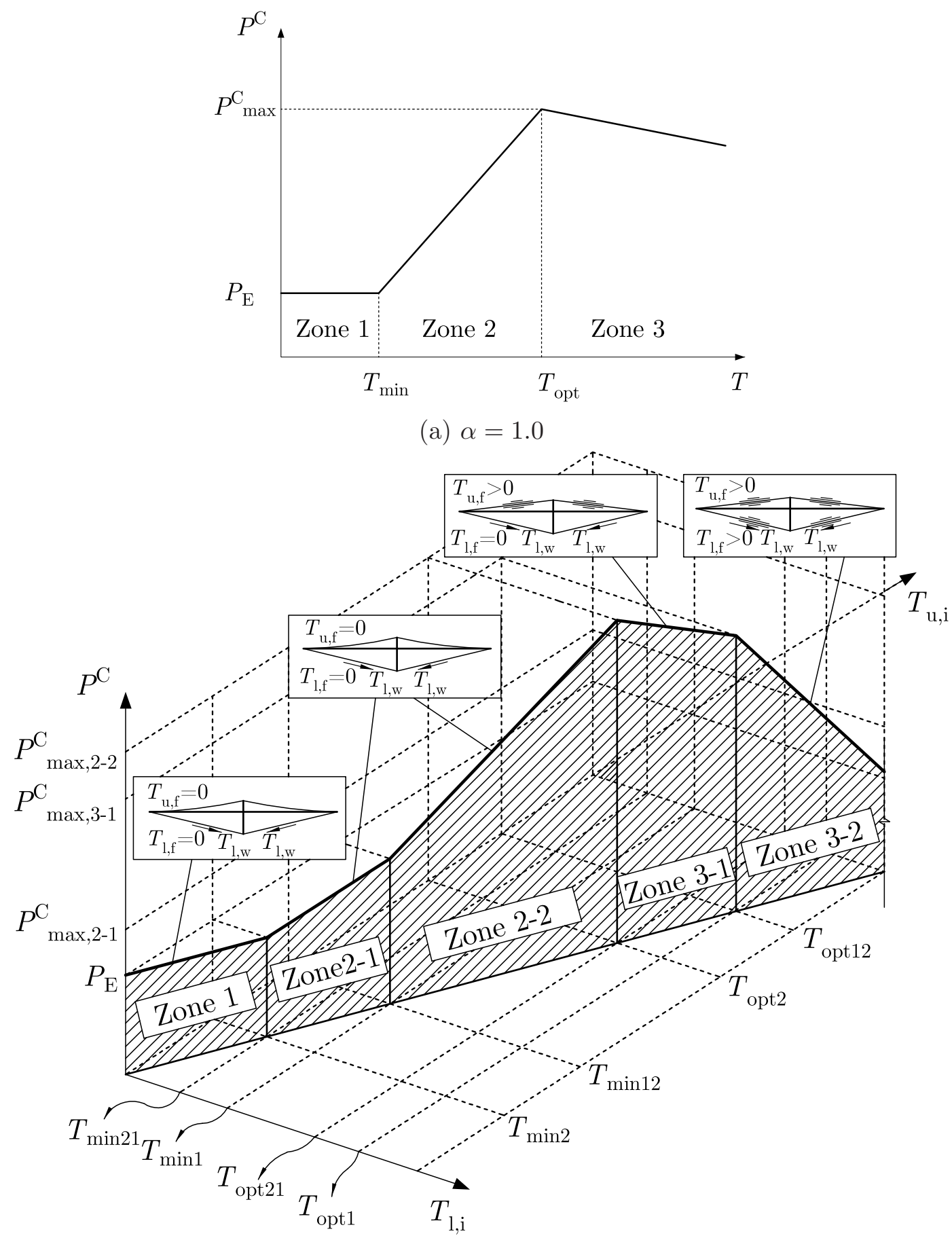

(b) $\alpha<1.0$

Figure 5: Zones of behaviour for prestressed stayed beam-columns with (a) $\alpha=1.0$ and (b) $\alpha<1.0$ : the graph shows the critical buckling load of the beam-column $P^{\mathrm{C}}$ plotted against different combinations of initial prestressing forces within the upper and lower stays $\left(T_{\mathrm{u}, \mathrm{i}}\right.$ and $T_{1, \mathrm{i}}$ respectively). Note that this is a line rather than a surface since the relationship between $T_{\mathrm{u}, \mathrm{i}}$ and $T_{\mathrm{l}, \mathrm{i}}$ is fixed through Eq. (8), which was shown in [30] to maximize the structural performance approximately. 
Again, for the case where $\alpha=1.0$, there is no such subdivision of the zone and $T_{1, \mathrm{i}}=T_{\mathrm{u}, \mathrm{i}} \geqslant T_{\mathrm{opt}}$, and $P^{\mathrm{C}}<P_{\max }^{\mathrm{C}}$.

In [30], the prestressing forces marking the boundary between Zones 2 and $3, T_{\text {opt1 }}$, $T_{\mathrm{opt} 2}, T_{\mathrm{opt} 12}$ and $T_{\mathrm{opt} 21}$ were determined to be thus:

$$
T_{\mathrm{opt} 1}=P_{\max }^{\mathrm{C}} C_{\mathrm{l}}, \quad T_{\mathrm{opt} 2}=P_{\mathrm{max}}^{\mathrm{C}} C_{\mathrm{u}}, \quad T_{\mathrm{opt} 12}=\frac{T_{\mathrm{opt} 1} \sin \beta_{1}}{\sin \beta_{\mathrm{u}}}, \quad T_{\mathrm{opt} 21}=\frac{T_{\mathrm{opt} 2} \sin \beta_{\mathrm{u}}}{\sin \beta_{\mathrm{l}}},
$$

where the maximum critical buckling load $P_{\max }^{\mathrm{C}}$ is evaluated from the expression:

$$
P_{\max }^{\mathrm{C}}=\frac{P_{\mathrm{FE}, T=0}^{\mathrm{C}}}{C_{0}},
$$

with $P_{\mathrm{FE}, T=0}^{\mathrm{C}}$ being the elastic buckling load of the studied stayed column with zero prestress determined using linear buckling analysis. In the present study, this is obtained using the commercial FE code ABAQUS [40], where the expression for $C_{0}$, which is a reduction factor to $P_{\mathrm{FE}, T=0}^{\mathrm{C}}$ to account for the influence of the prestress, is given thus:

$$
C_{0}=1+\frac{1}{2 K_{\mathrm{c}, \mathrm{a}}}\left(\frac{K_{\mathrm{su}} K_{\mathrm{au}, \mathrm{a}} \cos ^{2} \beta_{\mathrm{u}}}{2 K_{\mathrm{su}} \sin ^{2} \beta_{\mathrm{u}}+K_{\mathrm{au}, \mathrm{a}}}+\frac{K_{\mathrm{sl}} K_{\mathrm{al}, \mathrm{a}} \cos ^{2} \beta_{\mathrm{l}}}{2 K_{\mathrm{sl}} \sin ^{2} \beta_{\mathrm{l}}+K_{\mathrm{al}, \mathrm{a}}}\right)
$$

with the quantities $C_{\mathrm{u}}=C_{1} / C_{3}$ and $C_{1}=C_{2} / C_{3}$, and the expressions for $C_{1}, C_{2}$ and $C_{3}$ being thus:

$$
\begin{aligned}
C_{1}= & K_{\mathrm{au}, \mathrm{a}} K_{\mathrm{su}}\left(2 K_{\mathrm{sl}} \sin ^{2} \beta_{\mathrm{l}}+K_{\mathrm{al}, \mathrm{a}}\right) \cos \beta_{\mathrm{u}} \\
C_{2}= & K_{\mathrm{al}, \mathrm{a}} K_{\mathrm{sl}}\left(2 K_{\mathrm{su}} \sin ^{2} \beta_{\mathrm{u}}+K_{\mathrm{au}, \mathrm{a}}\right) \cos \beta_{1} \\
C_{3}=8 & K_{\mathrm{c}, \mathrm{a}} K_{\mathrm{sl}} K_{\mathrm{su}} \sin ^{2} \beta_{1} \sin ^{2} \beta_{\mathrm{u}}+2 K_{\mathrm{al}, \mathrm{a}} K_{\mathrm{sl}} K_{\mathrm{su}} \cos ^{2} \beta_{\mathrm{l}} \sin ^{2} \beta_{\mathrm{u}} \\
& +2 K_{\mathrm{au}, \mathrm{a}} K_{\mathrm{sl}} K_{\mathrm{su}} \cos ^{2} \beta_{\mathrm{u}} \sin ^{2} \beta_{\mathrm{l}}+4 K_{\mathrm{c}, \mathrm{a}} K_{\mathrm{al}, \mathrm{a}} K_{\mathrm{su}} \sin ^{2} \beta_{\mathrm{u}}+4 K_{\mathrm{c}, \mathrm{a}} K_{\mathrm{au}, \mathrm{a}} K_{\mathrm{sl}} \sin ^{2} \beta_{\mathrm{l}} \\
& \quad+K_{\mathrm{al}, \mathrm{a}} K_{\mathrm{au}, \mathrm{a}} K_{\mathrm{sl}} \cos ^{2} \beta_{\mathrm{l}}+K_{\mathrm{al}, \mathrm{a}} K_{\mathrm{au}, \mathrm{a}} K_{\mathrm{su}} \cos ^{2} \beta_{\mathrm{u}}+2 K_{\mathrm{c}, \mathrm{a}} K_{\mathrm{al}, \mathrm{a}} K_{\mathrm{au}, \mathrm{a}} .
\end{aligned}
$$

\section{Prestress and pre-cambering}

In [30] and [33], the mid-span displacement after step 3 was assumed to be zero by satisfying Eq. (8), which was derived on the assumption of linear behaviour. However, owing to the influence of geometric nonlinearity, namely the effect of the beam-column shortening in length due to introduction of prestress, an effect that is more pronounced for the mono-symmetric systems, the mid-span vertical displacement is in fact non-zero. This non-zero mid-span displacement essentially provides pre-cambering; the distribution of prestressing forces between upper and lower stays can be altered deliberately to achieve desired levels of pre-cambering (indicated by the mid-span displacement after applying the prestress in step 3, i.e. $w_{\mathrm{p}, \mathrm{m}}$ ) and affect the buckling and post-buckling behaviour in step 4. The principal purpose of the current section is to investigate the relationship between the distribution of the stay prestress and the level of pre-cambering. The FE models in the present section are perfect without any initial geometric imperfections. 
To study the effect on the pre-cambering from prestressing, a pre-cambering index $I_{\mathrm{p}}$ is defined, thus:

$$
I_{\mathrm{p}}=10000 \frac{w_{\mathrm{p}, \mathrm{m}}}{L},
$$

where $w_{\mathrm{p}, \mathrm{m}}$ is the mid-span displacement after introducing the prestress in step 3 . The cases where $I_{\mathrm{p}}=\{-5,-1,0,1,5\}$ are adopted as the benchmark pre-cambering levels. Positive values of $I_{\mathrm{p}}$ and $w_{\mathrm{p}, \mathrm{m}}$ represent upward pre-cambering, while negative values correspond to downward pre-cambering. The distribution of prestressing forces between the upper and lower stays is represented by a newly defined parameter $\gamma$, thus:

$$
\gamma=\frac{T_{\mathrm{u}, \mathrm{i}} \sin \beta_{\mathrm{u}}}{T_{\mathrm{l}, \mathrm{i}} \sin \beta_{\mathrm{l}}}
$$

which is a ratio of the applied prestressing level to the first order prestressing level from Eq. (8). For the instance where $\gamma=1$, Eqs. (8) and (14) are of course identical.

\subsection{Doubly-symmetric cases}

Doubly-symmetric cases $(\alpha=1)$ have upper and lower crossarms of identical length. Changes to the pre-cambering index $I_{\mathrm{p}}$ at $T_{1, \mathrm{i}}=T_{\mathrm{opt} 1}$ and $T_{1, \mathrm{i}}=2 T_{\mathrm{opt} 1}$ with increasing $\gamma$ for cases $\mathrm{F} 1$ and A1, F2-F6, and A2-A6 are shown respectively in Figures 6, 7 and 8 .

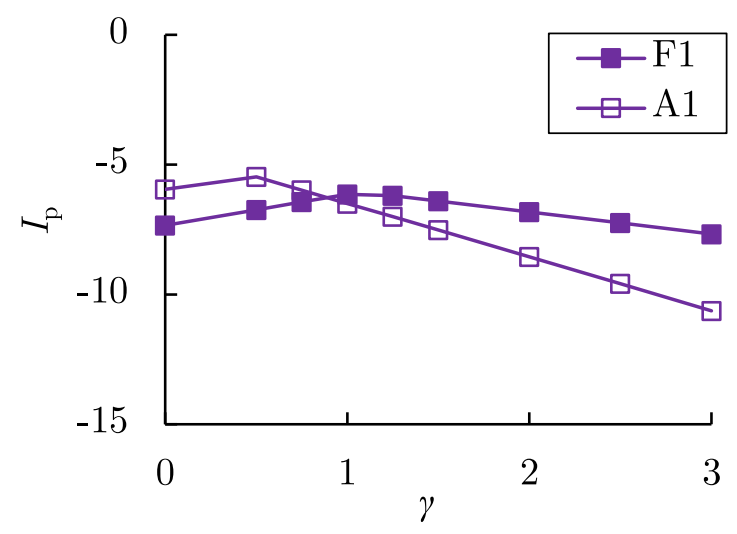

(a) $T_{1, \mathrm{i}}=T_{\mathrm{opt} 1}$

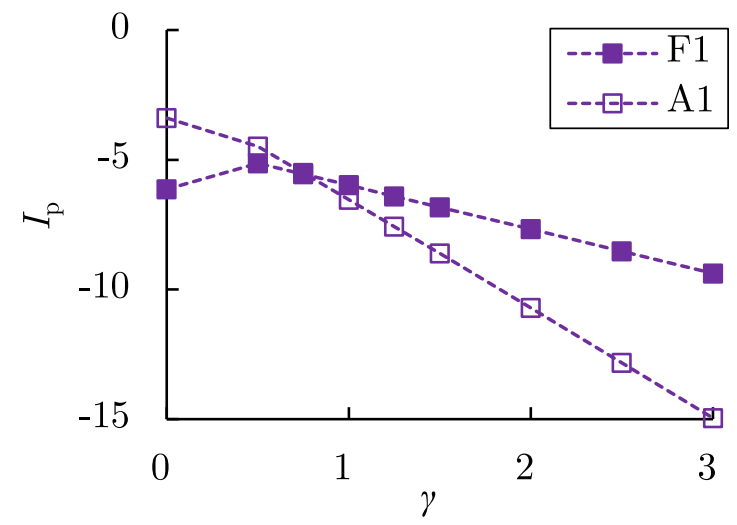

(b) $T_{1, \mathrm{i}}=2 T_{\mathrm{opt} 1}$

Figure 6: Pre-cambering versus prestressing distribution for cases F1 and A1.

It can be seen that the results in Figure 6 are significantly different from the results in Figures 7 and 8, implying that pre-cambering with $I_{\mathrm{p}} \geqslant 0$, cannot be achieved by adjusting the prestressing distribution between the upper and lower stays while $T_{1, \mathrm{i}} \leqslant 2 T_{\mathrm{opt} 1}$. This is because the influence of the stays and crossarms are limited in these cases owing to the small stay diameter $\phi_{\mathrm{s}}$ in case F1 and the small crossarm length $a_{1}$ in case A1. In addition, considering the low load-carrying capacity of these two systems from [30, 33], small stay diameters (i.e. $\phi_{\mathrm{s}} /\left(2 r_{\mathrm{c}}\right)<0.14$ corresponding to $\phi_{\mathrm{s}}<3.2 \mathrm{~mm}$ currently) and crossarm lengths (i.e. $\alpha_{1}<0.05$ ) should be avoided. However, for cases F2-F6 and A2-A6, a deliberate pre-cambering level, within the range of $I_{\mathrm{p}}=[-5,5]$, can be achieved for most 


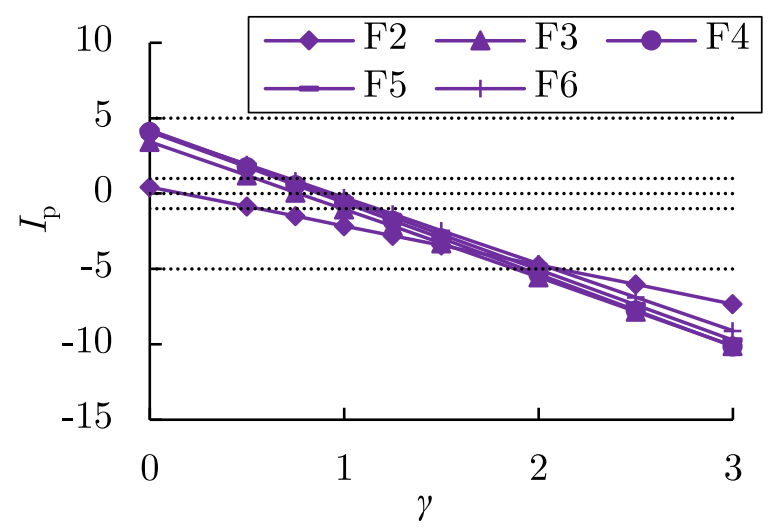

(a) $T_{1, \mathrm{i}}=T_{\mathrm{opt} 1}$

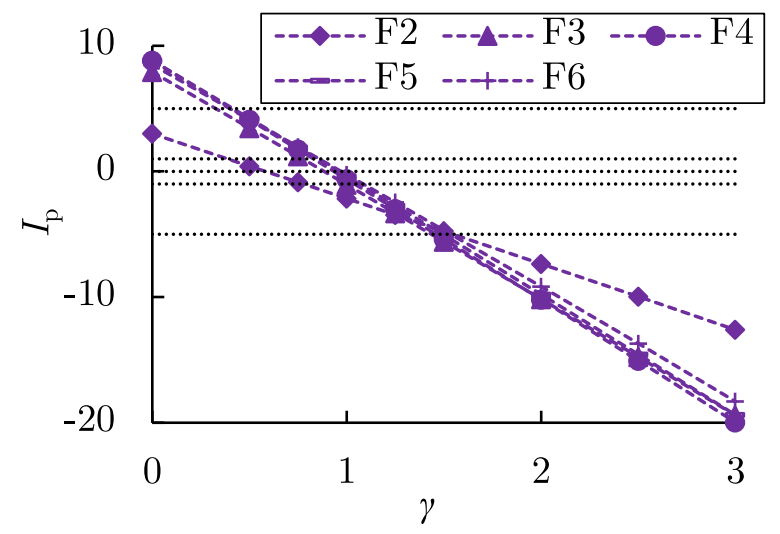

(b) $T_{1, \mathrm{i}}=2 T_{\mathrm{opt} 1}$

Figure 7: Pre-cambering versus prestressing distribution for cases F2-F6. The dotted horizontal lines from bottom to top correspond to $I_{\mathrm{p}}=\{-5,-1,0,1,5\}$ respectively.

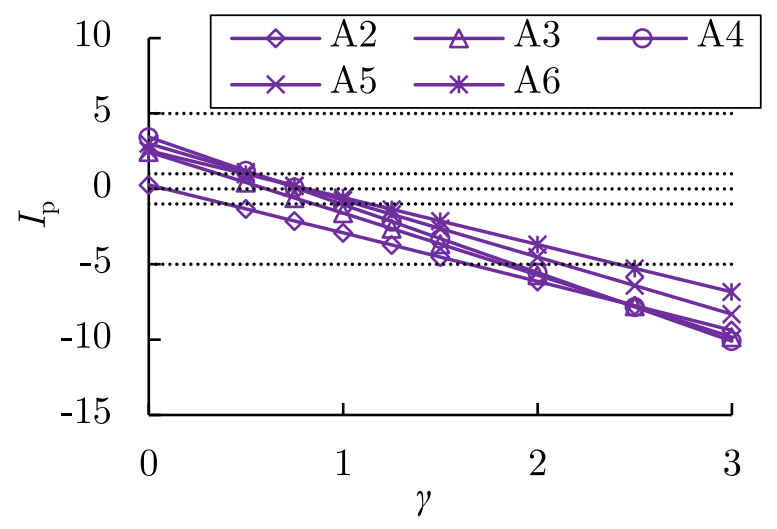

(a) $T_{1, \mathrm{i}}=T_{\text {opt1 }}$

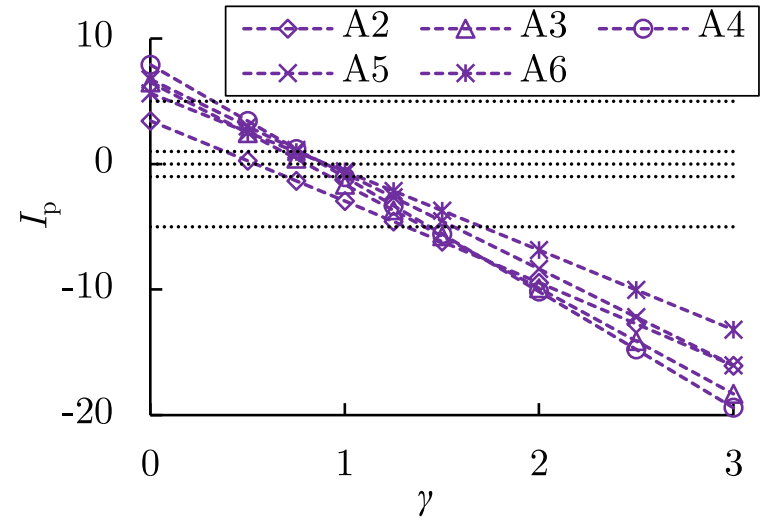

(b) $T_{1, \mathrm{i}}=2 T_{\mathrm{opt} 1}$

Figure 8: Pre-cambering versus prestressing distribution for cases A2-A6. The dotted horizontal lines from bottom to top correspond to $I_{\mathrm{p}}=\{-5,-1,0,1,5\}$ respectively. 
of these ten cases. The buckling and post-buckling behaviour of these ten cases will be investigated further in Section 4.

\subsection{Mono-symmetric cases}

Distinct from the doubly-symmetric cases, pre-cambering is more significant in monosymmetric cases because the actual (nonlinear) behaviour deviates more significantly from Eq. (8) than in the doubly-symmetric cases, because when $\alpha<1.0$ an upward force is introduced due to the end-shortening in the main member due to the prestressing.

Changes to the pre-cambering index $I_{\mathrm{p}}$ with increasing $\gamma$ at $T_{1, \mathrm{i}}=T_{\mathrm{opt} 1}$ for cases F3.1F3.4, F3.5-F3.7, and F3.8, F3.9 and F3 are shown respectively in Figures 9, 10 and 11.

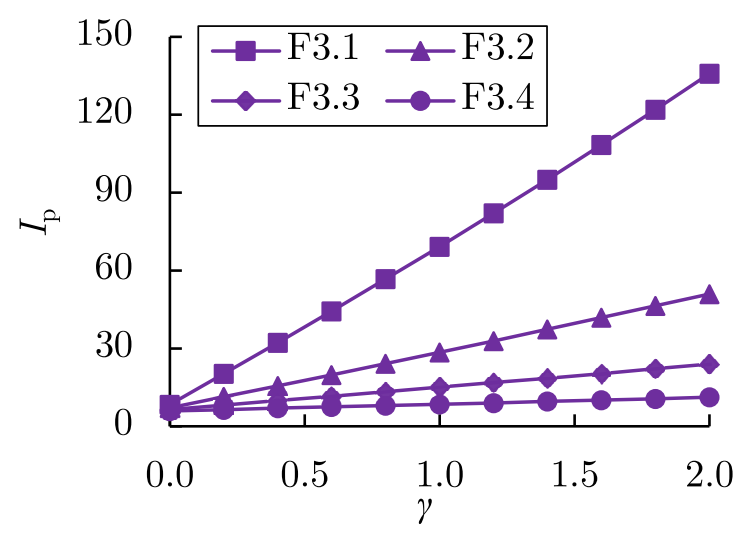

(a) $T_{1, \mathrm{i}}=T_{\mathrm{opt} 1}$

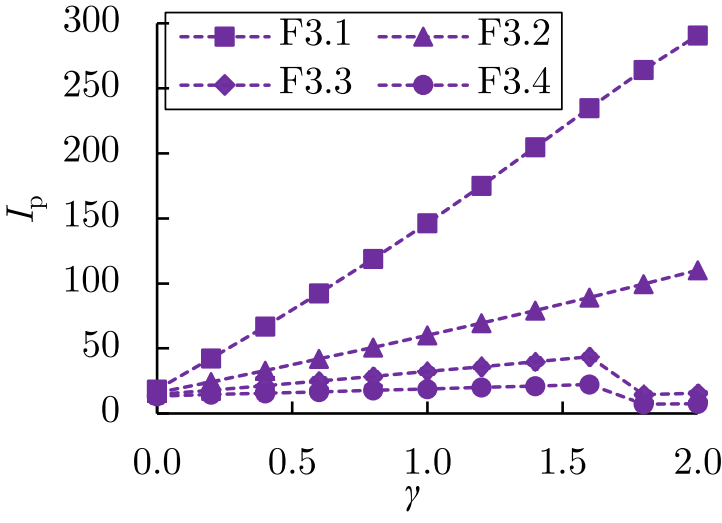

(b) $T_{1, \mathrm{i}}=2 T_{\text {opt } 1}$

Figure 9: Pre-cambering versus prestressing distribution for cases F3.1-F3.4.

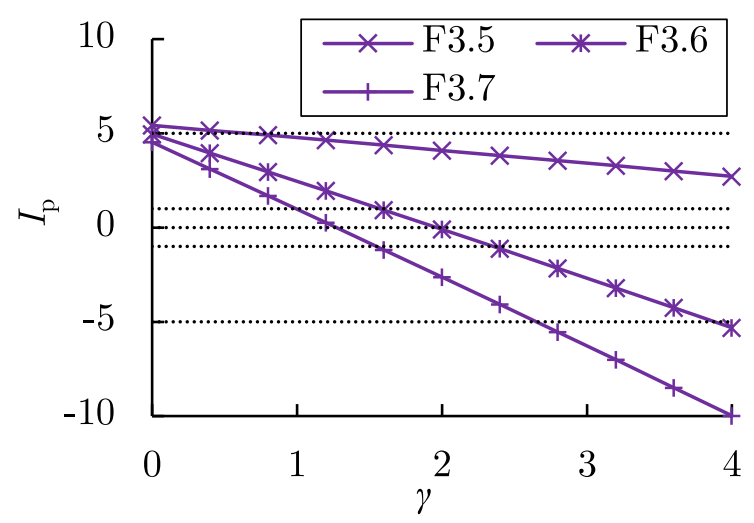

(a) $T_{1, \mathrm{i}}=T_{\mathrm{opt} 1}$

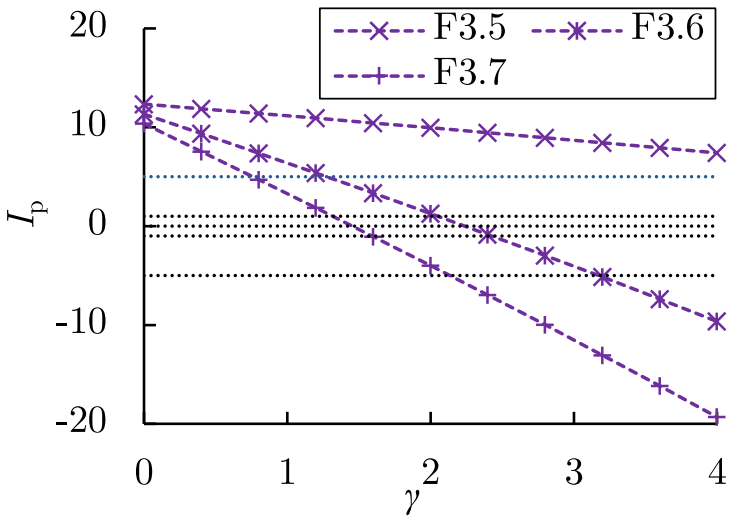

(b) $T_{1, \mathrm{i}}=2 T_{\text {opt1 }}$

Figure 10: Pre-cambering versus prestressing distribution for cases F3.5-F3.7. The dotted horizontal lines from bottom to top correspond to $I_{\mathrm{p}}=\{-5,-1,0,1,5\}$ respectively. Note that case F3.5 only crosses $I_{\mathrm{p}}=5$ in (a).

From Figure 9, for cases F3.1-F3.4, it can be seen that the downward pre-cambering 


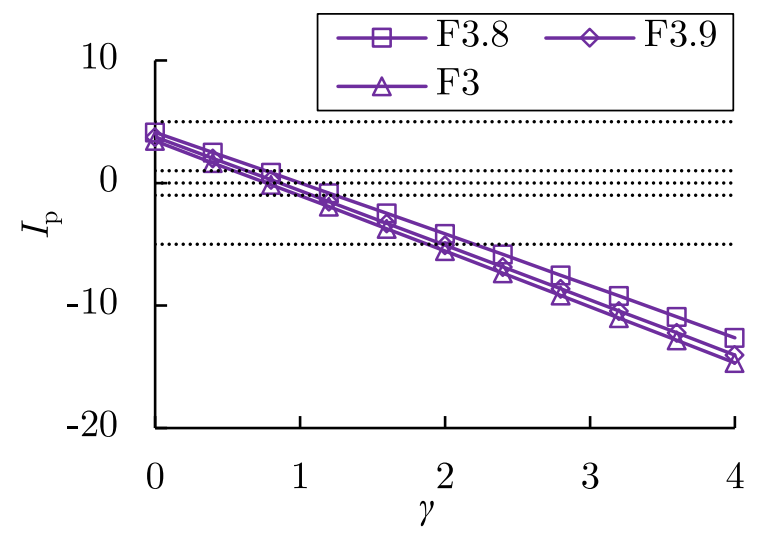

(a) $T_{1, \mathrm{i}}=T_{\mathrm{opt} 1}$

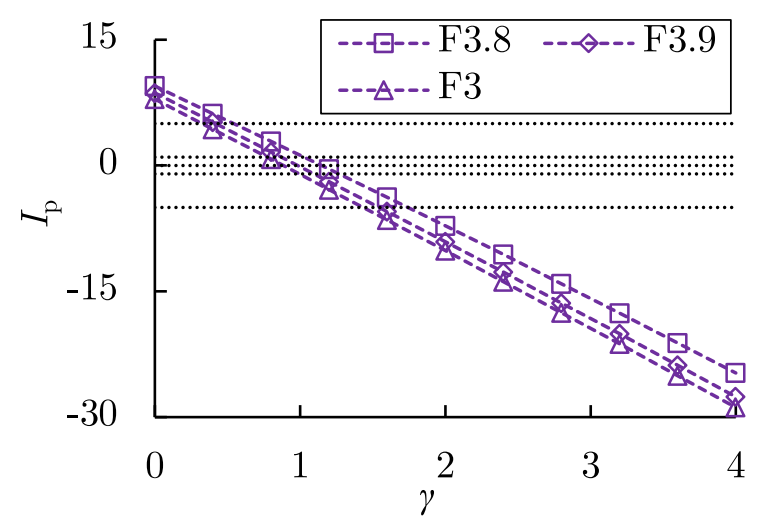

(b) $T_{1, \mathrm{i}}=2 T_{\mathrm{opt} 1}$

Figure 11: Pre-cambering versus prestressing distribution for cases F3.8, F3.9 and F3. The dotted horizontal lines from bottom to top correspond to $I_{\mathrm{p}}=\{-5,-1,0,1,5\}$ respectively.

with $I_{\mathrm{p}} \leqslant 0$ cannot be achieved by adjusting the prestressing distribution. Considering the unstable post-buckling behaviour of these four cases, as determined in [30, 33], small crossarm length ratios (i.e. $\alpha<0.5$ ) are best avoided. From Figures 10 and 11, for cases F3.5-F3.9 and F3, it can be seen that with increasing $\gamma, I_{\mathrm{p}}$ decreases consistently from positive to negative values. It implies that adjusting the distribution of prestress between the upper and lower stays allows desired pre-cambering levels to be obtained, regardless of the initial member shapes with upward and downward pre-cambering. It is observed that for cases F3.8, F3.9 and F3, the gradients of their relationships are similar, while for cases F3.5-F3.7, the gradients of their relationships are significantly different.

Finally, it should be stressed that the analysis performed in this subsection purely shows the pre-cambering levels achieved after applying varying levels of prestress in step 3. In Section 4, the structural performance of stayed beam-columns with three specific pre-cambering levels $\left(I_{\mathrm{p}}=\{-5,0,5\}\right)$ is studied.

\section{Pre-cambering, imperfection and prestressing sensitivities}

The principal purpose of the current section is to investigate the buckling and postbuckling behaviour of imperfect stayed beam-columns under axial compression in conjunction with self-weight using FE modelling. Following the recent advances in understanding relating to the zonal behaviour [30] and mode interaction [33] in perfect stayed beamcolumns under the same loading case as studied herein, the nonlinear behaviour considering different pre-cambering levels, imperfections, and prestressing forces is now examined.

A geometric imperfection amplitude ratio $\delta$ of $1 / 1000$ is adopted unless otherwise stated. The imperfection combinations in Table 4 are adopted to trigger the different buckling modes, including interactive buckling. In terms of the study on the sensitivity to varying imperfection amplitudes, combination 3 is adopted as the imperfection profile for cases F3 and F3.6. Owing to self-weight and pre-cambering from prestress, the principal deflection 
direction of the Mode 1 imperfection needs to be considered. A downward imperfection (i.e. the same as the self-weight direction) is amplified by the self-weight, and an upward imperfection (i.e. opposing the self-weight direction) is reduced by the self-weight. Although the displacement of the mid-span point is fixed after adjusting the prestressing distribution for a specific $I_{\mathrm{p}}$ value in step 3, the remainder of the member still deflects due to its self-weight. In terms of the studies on the sensitivity to varying imperfection combinations and prestressing levels, only the imperfections with the downward principal deflection (combined Mode 2 and downward Mode 1 imperfections) are adopted to trigger interactive buckling, since it was demonstrated in [33] that mode interaction only occurs in the downward buckling mode.

\section{1. $I_{\mathrm{p}}=-5$}

Figure 12 shows the ultimate loads for cases F3 and F3.6 with varying imperfection

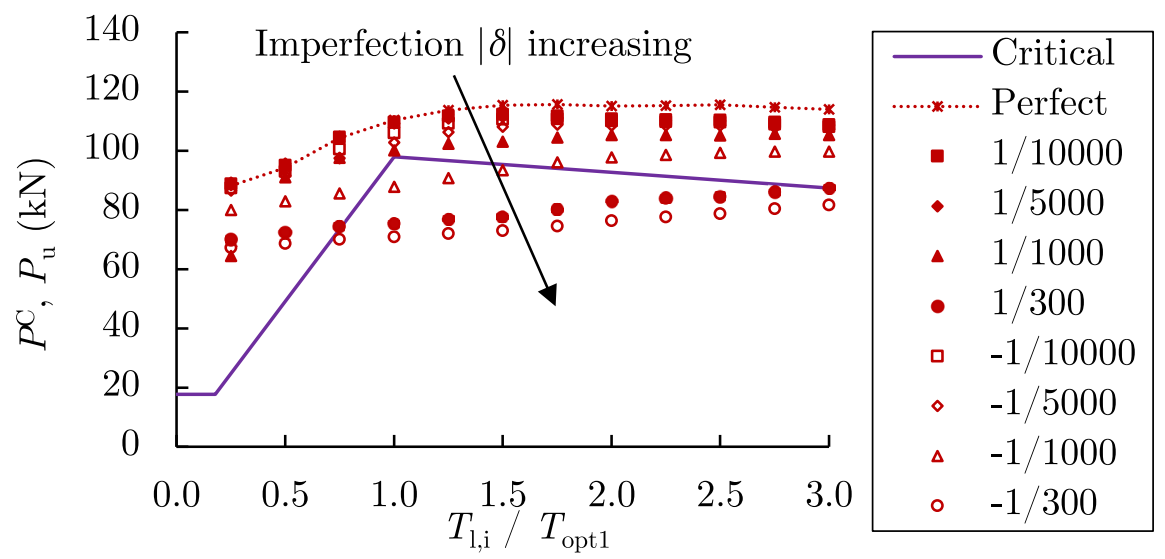

(a) F3

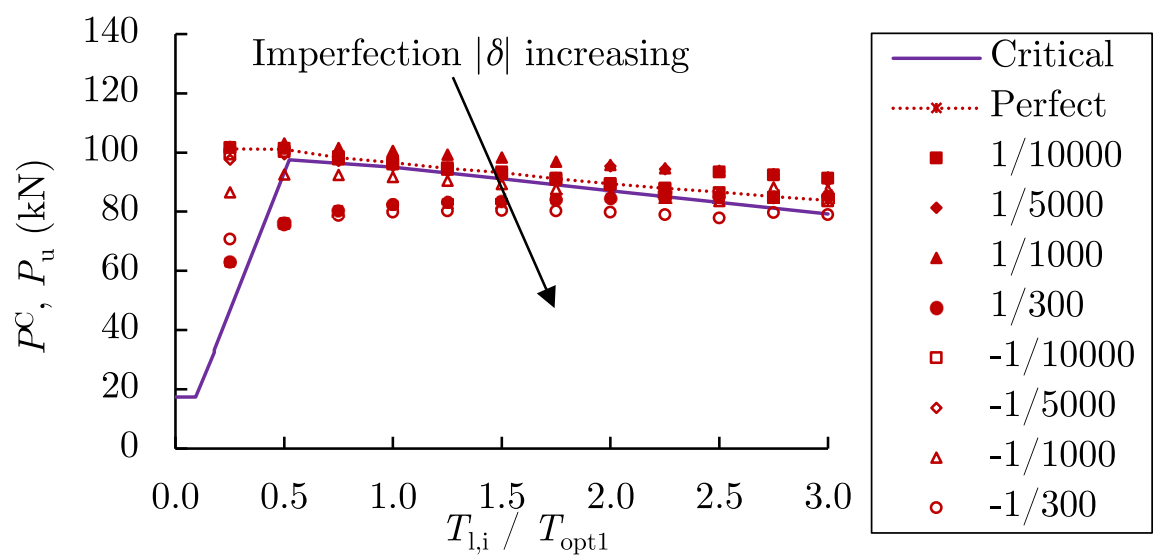

(b) F3.6

Figure 12: Critical buckling (solid line) and ultimate loads (all symbols) for cases F3 and F3.6 with a pre-cambering index $I_{\mathrm{p}}=-5$ and varying imperfection amplitudes $\delta ; P^{\mathrm{C}}$ is the critical buckling load from the analytically-defined zones [30]; $P_{\mathrm{u}}$ is the ultimate load from FE modelling. 
amplitudes and the pre-cambering index $I_{\mathrm{p}}=-5$. It can be seen that for both cases F3 and F3.6, the load-carrying capacity decreases with increasing imperfection amplitudes, but for case F3.6 the reduction is less significant than that for case F3. Compared to the imperfections with the upward principal deflection, the imperfections with the downward principal deflection lead to lower ultimate loads, as might be expected. In addition, for case F3.6, small imperfections in the upward principal deflection can in fact increase the ultimate load slightly.

Figure 13 shows the ultimate loads for the different imperfection combinations with

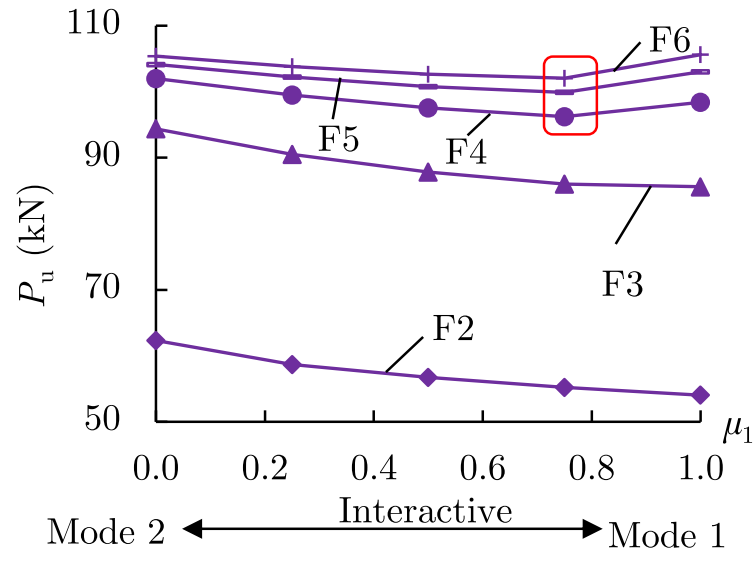

(a) F2-F6

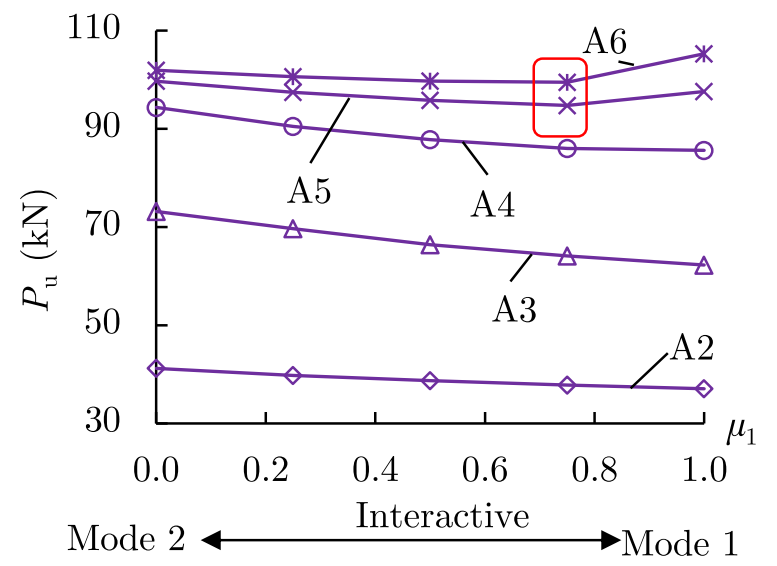

(b) A2-A6

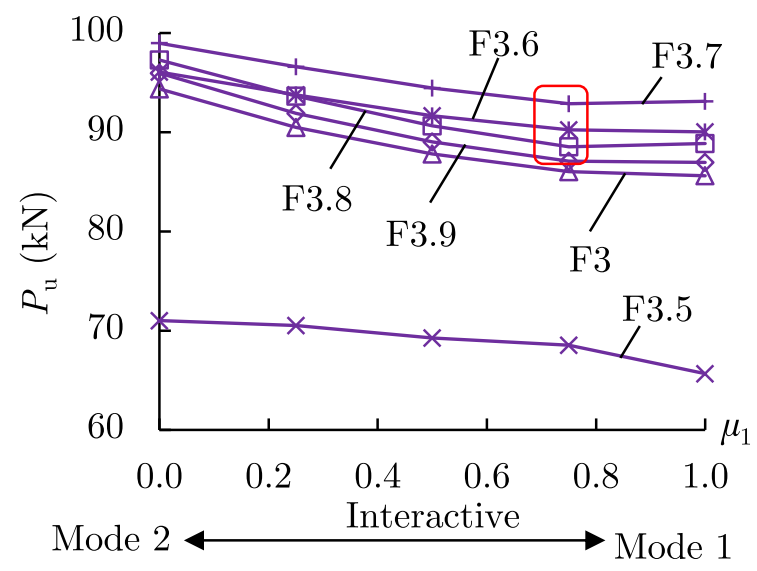

(c) F3.5-F3.9 and F3

Figure 13: Sensitivity to the imperfection combinations represented by $P_{\mathrm{u}}$ versus $\mu_{1}$ with $I_{\mathrm{p}}=-5$ and (a) varying stay diameter $\phi_{\mathrm{s}}$, (b) varying lower crossarm length $a_{1}$ and (c) varying crossarm length ratio $\alpha$. The encircled cases denote the minimum ultimate loads for the respective configurations.

$I_{\mathrm{p}}=-5$ and a variation of the parameters $\phi_{\mathrm{s}}, a_{\mathrm{l}}$ and $\alpha$ at $T_{1, \mathrm{i}}=T_{\mathrm{opt} 1}$. For the doublysymmetric cases, F2-F6 and A2-A6, as can be seen, when Mode 1 is critical, the lowest ultimate load is seen within the Mode 1 buckling case. However, when Mode 2 buckling is critical, the lowest ultimate load is always seen within the interactive buckling case, such as cases F4-F6 and A5-A6. For the mono-symmetric cases, interactive buckling becomes 
dominant in cases F3.6-F3.8 that have intermediate values of the crossarm length ratio $\alpha$. Moreover, for all cases, there are only small differences within the interactive buckling region regardless of the different values of $\mu_{1}$, i.e. different combinations of $\mu_{1}$ and $\mu_{2}$. This result indicates that interactive buckling behaviour is not very sensitive to the combination of the combined imperfection profiles. These conclusions mirror those from the previous study on vertically stayed columns [18].

The ultimate loads for stayed beam-columns at $T_{1, \mathrm{i}}=T_{\mathrm{opt} 1}$ and $T_{1, \mathrm{i}}=2 T_{\mathrm{opt} 1}$ with $I_{\mathrm{p}}=-5$ and varying values of $\phi_{\mathrm{s}}, a_{\mathrm{l}}$ and $\alpha$ are shown in Figures 14, 15, and 16. From

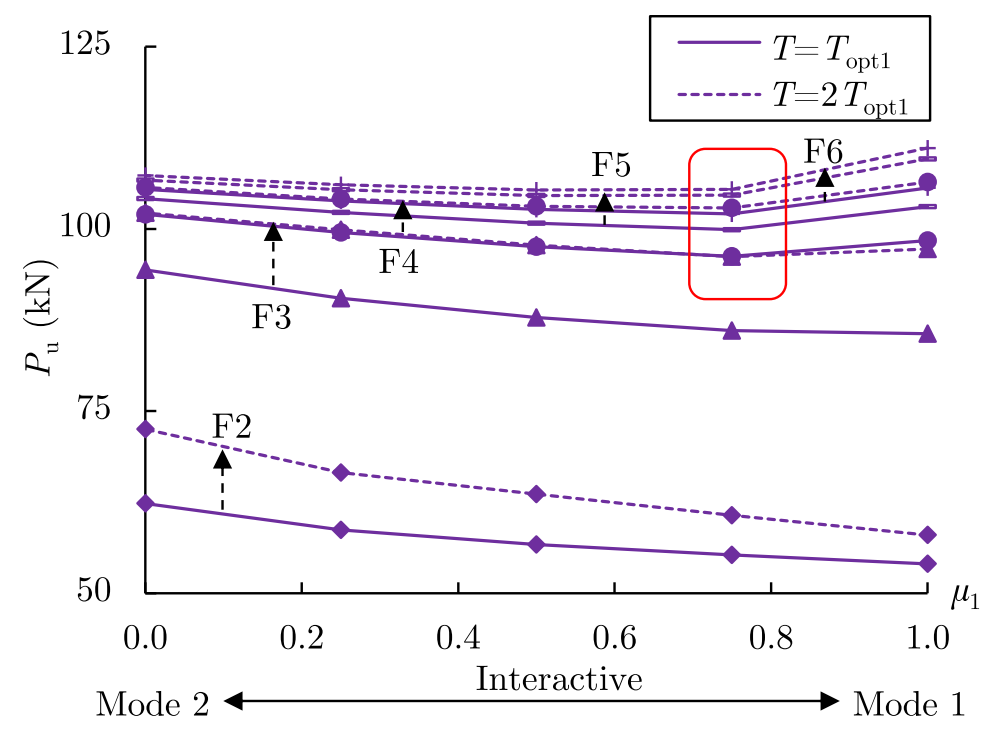

Figure 14: Sensitivity to the downward imperfection combinations represented by $P_{\mathrm{u}}$ versus $\mu_{1}$ with $I_{\mathrm{p}}=-5$ and varying stay diameter $\phi_{\mathrm{s}}$ at $T_{\mathrm{l}, \mathrm{i}}=T_{\mathrm{opt} 1}$ and $T_{\mathrm{l}, \mathrm{i}}=2 T_{\mathrm{opt} 1}$. The arrows with the dashed lines denote an increase or decrease in the ultimate load from $T_{\mathrm{opt} 1}$ to $2 T_{\mathrm{opt} 1}$.

Figures 14 and 15, for the doubly-symmetric cases, increasing the prestressing force results in a rise in the ultimate load for each imperfection combination. Although the maximum critical buckling load can be seen at $T_{1, \mathrm{i}}=T_{\mathrm{opt} 1}$ from linear analysis [30], $T_{\mathrm{opt} 1}$ does not maximize the ultimate load, and introducing a prestressing force that is higher than this level is recommended in practice. From Figure 16, for the mono-symmetric cases F3.5F3.7, increasing the prestressing levels reduces the ultimate load, while for other cases with larger $\alpha$, i.e. cases F3.8, F3.9 and F3, the load-carrying capacity increases significantly from $T_{1, \mathrm{i}}=T_{\mathrm{opt} 1}$ to $T_{1, \mathrm{i}}=2 T_{\mathrm{opt} 1}$. In addition, for cases F3, A4, F3.9 and F3, at $T_{1, \mathrm{i}}=T_{\mathrm{opt} 1}$, interactive buckling does not dominate the behaviour, while for $T_{1, \mathrm{i}}=2 T_{\mathrm{opt} 1}$, interactive buckling is dominant.

\section{2. $I_{\mathrm{p}}=0$}

Figure 17 shows the ultimate loads for cases F3 and F3.6 with varying imperfection amplitudes and the pre-cambering index $I_{\mathrm{p}}=0$. For case F3, the difference of ultimate loads with imperfections in the different principal directions is small. However, for case 


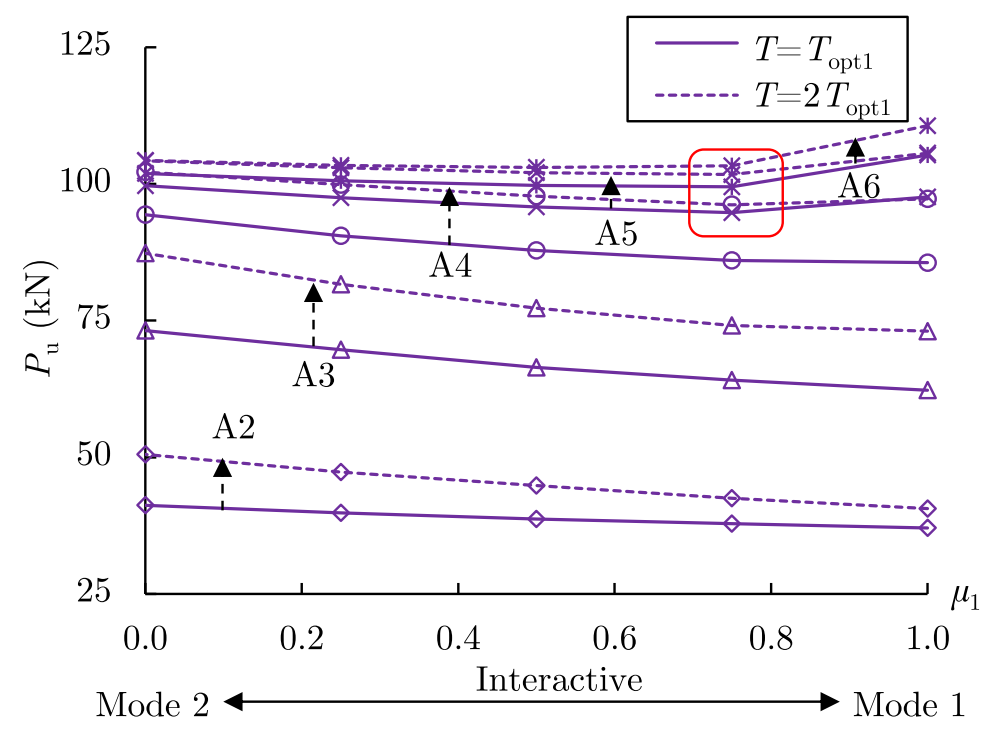

Figure 15: Sensitivity to the downward imperfection combinations represented by $P_{\mathrm{u}}$ versus $\mu_{1}$ with $I_{\mathrm{p}}=-5$ and varying lower crossarm length $a_{\mathrm{l}}$ at $T_{\mathrm{l}, \mathrm{i}}=T_{\mathrm{opt} 1}$ and $T_{\mathrm{l}, \mathrm{i}}=2 T_{\mathrm{opt} 1}$. Axes and labels are the same as Figure 14.

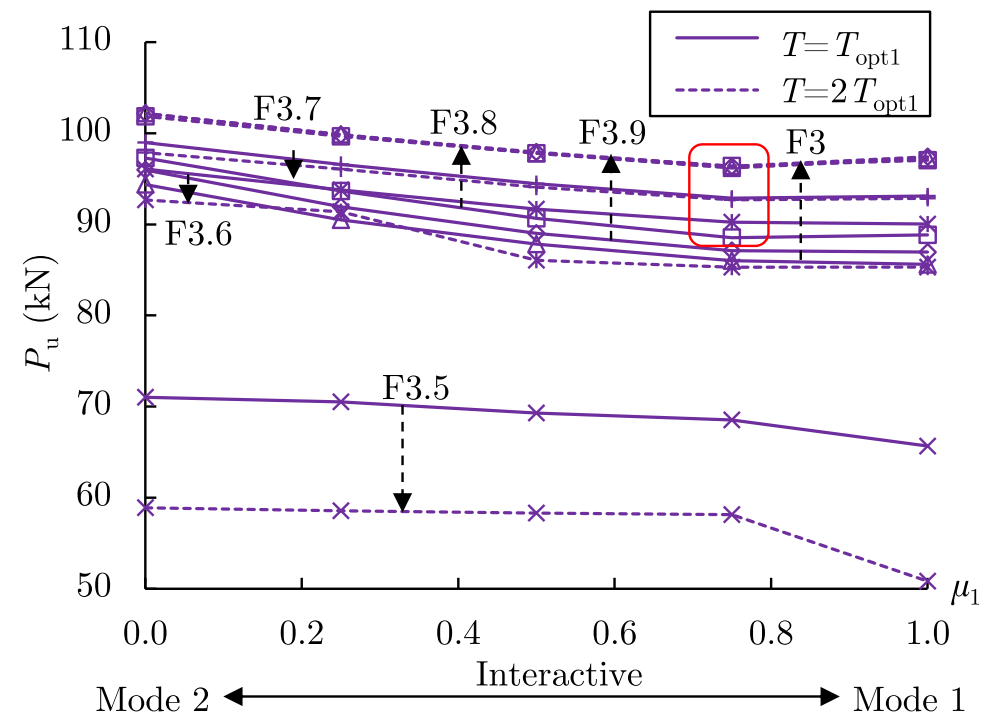

Figure 16: Sensitivity to the downward imperfection combinations represented by $P_{\mathrm{u}}$ versus $\mu_{1}$ with $I_{\mathrm{p}}=-5$ and varying crossarm length ratio $\alpha$ at $T_{1, \mathrm{i}}=T_{\mathrm{opt} 1}$ and $T_{1, \mathrm{i}}=2 T_{\mathrm{opt} 1}$. Axes and labels are the same as Figure 14. 


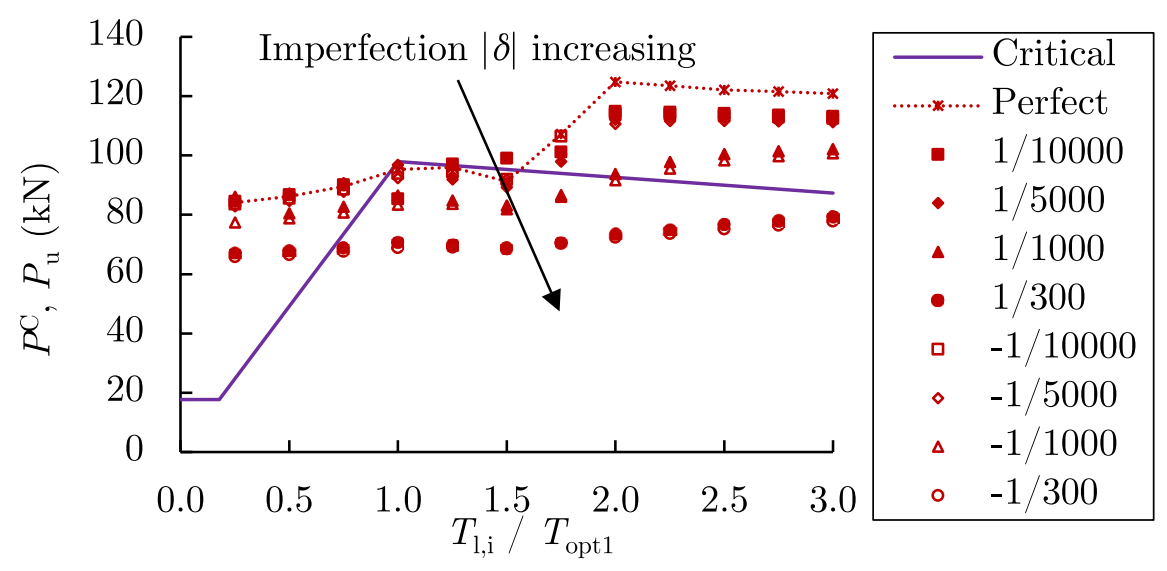

(a) F3

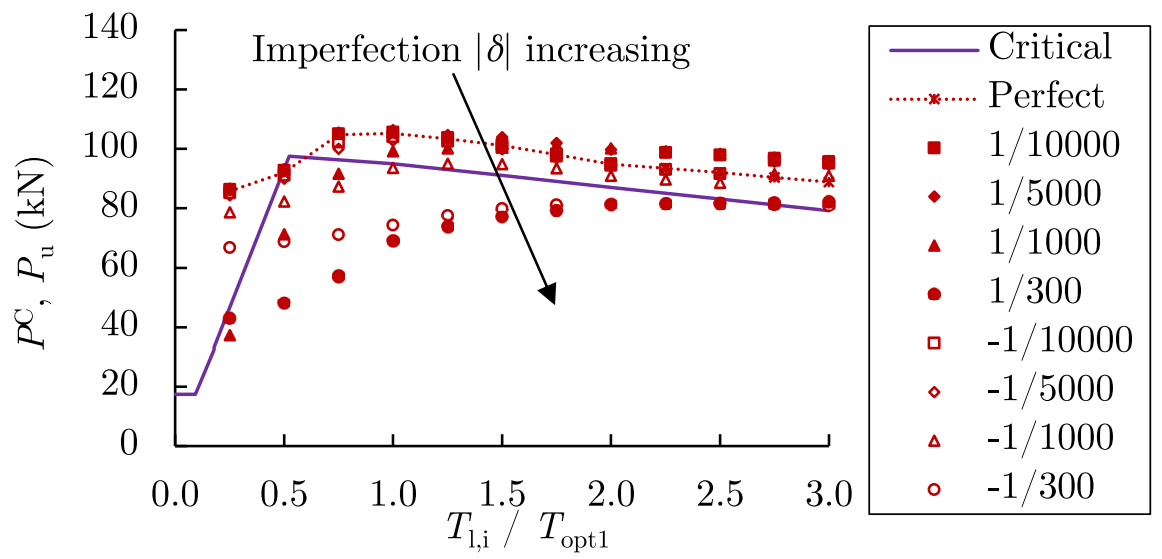

(b) F3.6

Figure 17: Critical buckling (solid line) and ultimate loads (all symbols) for cases F3 and F3.6 with $I_{\mathrm{p}}=0$ and varying $\delta$. Axes and labels are the same as Figure 12. 
F3.6, the reduction from the upward imperfections is more significant than that from the imperfections which are downwards.

Figure 18 shows the ultimate loads for the different imperfection combinations with

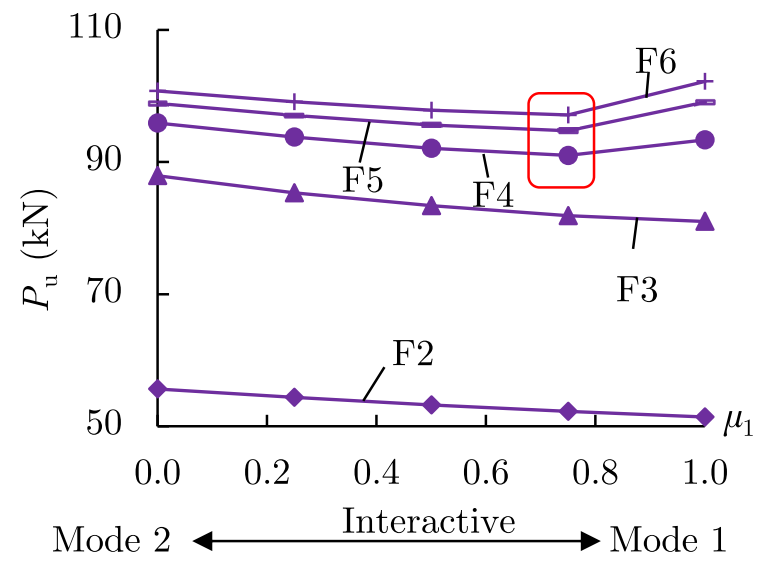

(a) F2-F6

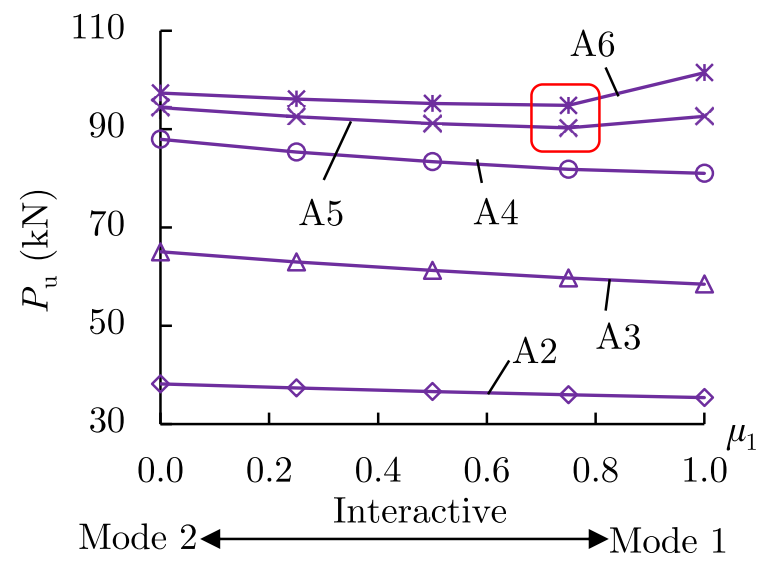

(b) A2-A6

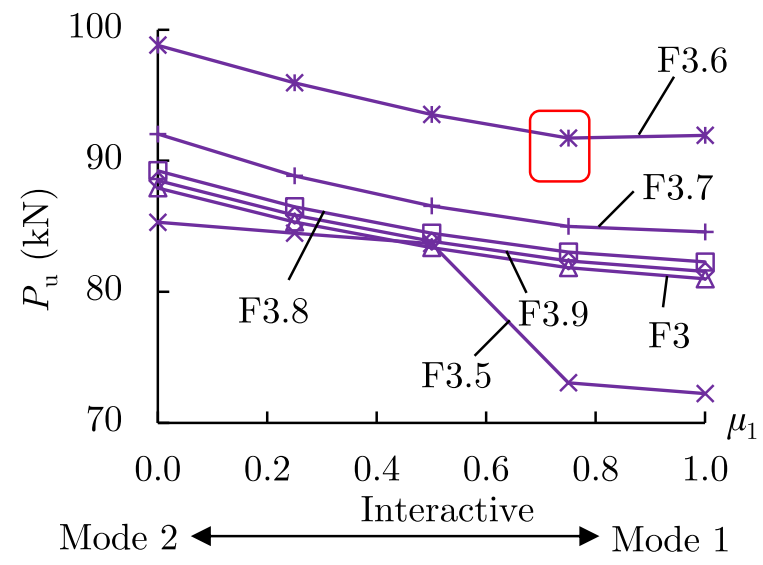

(c) F3.5-F3.9 and F3

Figure 18: Sensitivity to the imperfection combinations represented by $P_{\mathrm{u}}$ versus $\mu_{1}$ with $I_{\mathrm{p}}=0$ and (a) varying $\phi_{\mathrm{s}}$, (b) varying $a_{1}$ and (c) varying $\alpha$.

$I_{\mathrm{p}}=0$ and a variation of the parameters $\phi_{\mathrm{s}}, a_{\mathrm{l}}$ and $\alpha$ at $T_{1, \mathrm{i}}=T_{\mathrm{opt} 1}$. Comparing Figure 18 with 13, for the doubly-symmetric cases, the behaviour in these systems with this precambering level is similar to that with $I_{\mathrm{p}}=-5$, but the load-carrying capacity for the cases with $I_{\mathrm{p}}=0$ is lower than that with $I_{\mathrm{p}}=-5$. The interactive buckling dominance does not change with the pre-cambering level varying. For the mono-symmetric cases with $I_{\mathrm{p}}=0$, case F3.6 performs best, and the difference in load-carrying capacity between case F3.6 and the other cases is significant. For cases with $I_{\mathrm{p}}=-5$, case F3.7 performs best, and the difference of the load-carrying capacity among cases F3.6-F3.9 and F3 is not significant.

The ultimate loads for stayed beam-columns where $T_{1, \mathrm{i}}=T_{\mathrm{opt1}}$ and $T_{1, \mathrm{i}}=2 T_{\text {opt1 }}$ with $I_{\mathrm{p}}=0$ and varying values of $\phi_{\mathrm{s}}, a_{1}$ and $\alpha$ are shown in Figures 19, 20, and 21. Comparing 


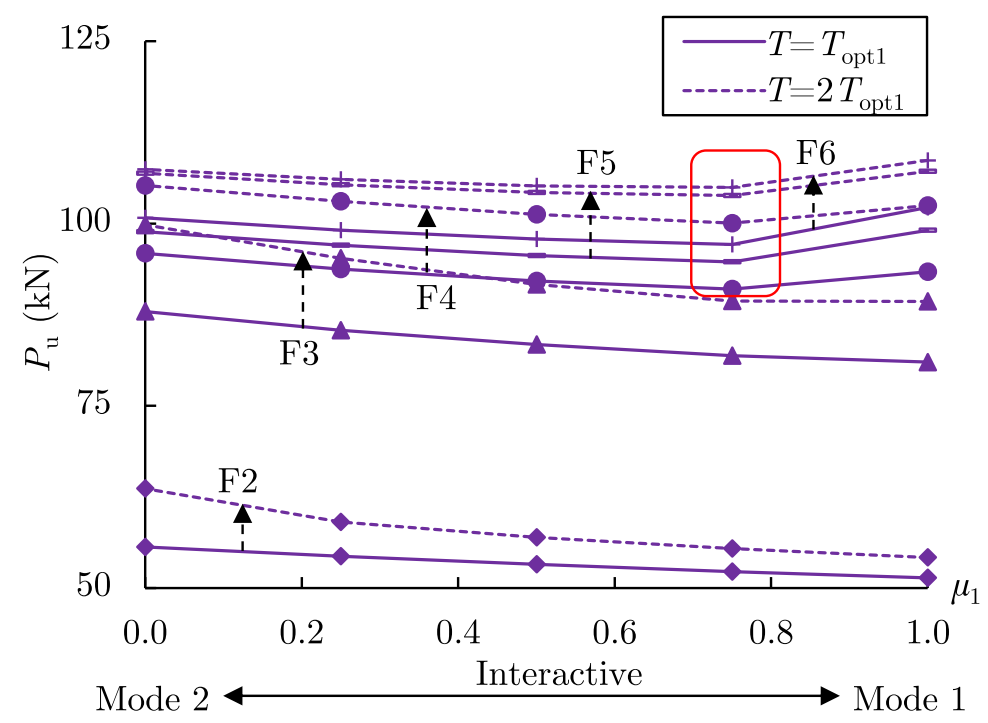

Figure 19: Sensitivity to the downward imperfection combinations represented by $P_{\mathrm{u}}$ versus $\mu_{1}$ with $I_{\mathrm{p}}=0$ and varying $\phi_{\mathrm{s}}$ at $T_{1, \mathrm{i}}=T_{\mathrm{opt} 1}$ and $T_{1, \mathrm{i}}=2 T_{\mathrm{opt} 1}$. Axes and labels are the same as Figure 14 .

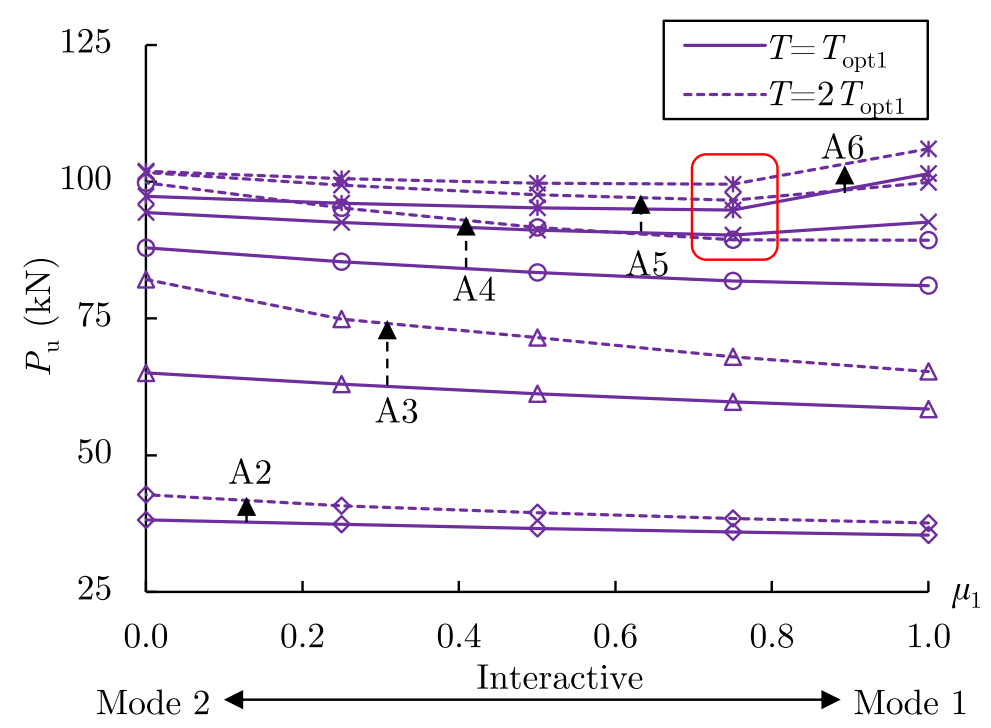

Figure 20: Sensitivity to the downward imperfection combinations represented by $P_{\mathrm{u}}$ versus $\mu_{1}$ with $I_{\mathrm{p}}=0$ and varying $a_{1}$ at $T_{1, \mathrm{i}}=T_{\mathrm{opt} 1}$ and $T_{1, \mathrm{i}}=2 T_{\mathrm{opt} 1}$. Axes and labels are the same as Figure 14 . 


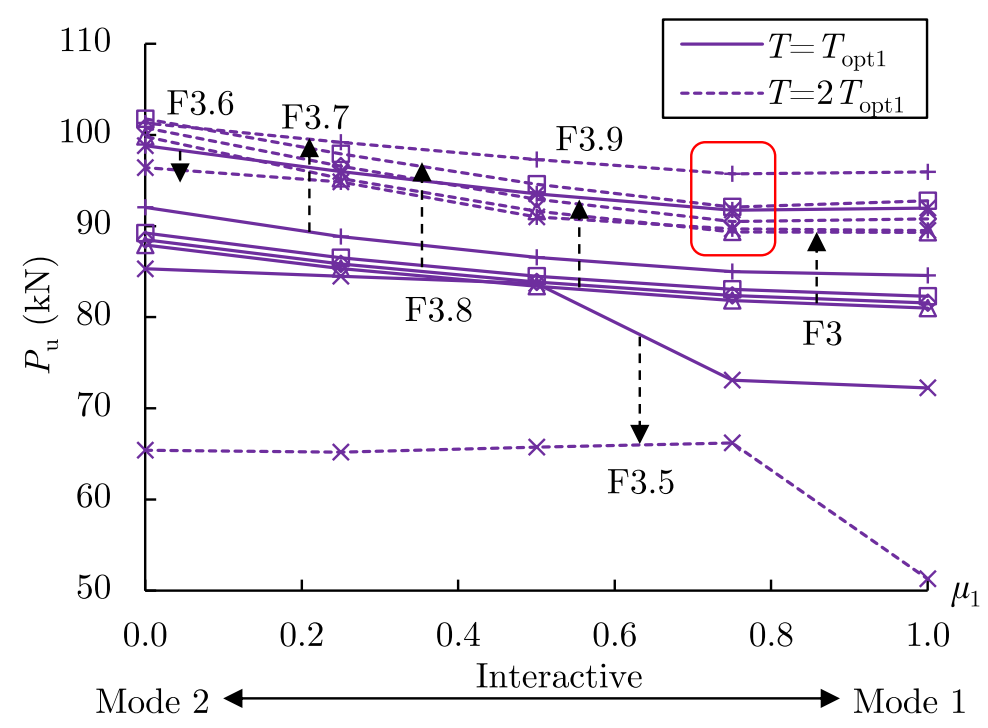

Figure 21: Sensitivity to the downward imperfection combinations represented by $P_{\mathrm{u}}$ versus $\mu_{1}$ with $I_{\mathrm{p}}=0$ and varying $\alpha$ at $T_{1, \mathrm{i}}=T_{\mathrm{opt} 1}$ and $T_{1, \mathrm{i}}=2 T_{\mathrm{opt} 1}$. Axes and labels are the same as Figure 14 .

Figures 19 and 20 with Figures 14 and 15, for the doubly-symmetric cases, similar to the results at $T_{1, \mathrm{i}}=T_{\mathrm{opt} 1}$, the behaviour in these systems with this pre-cambering level is similar to that with $I_{\mathrm{p}}=-5$, but their load-carrying capacity with $I_{\mathrm{p}}=0$ is lower than that with $I_{\mathrm{p}}=-5$. Comparing Figure 21 with Figure 16, for case F3.7 where $I_{\mathrm{p}}=0$, the loadcarrying capacity can be increased significantly by changing $T_{1, \mathrm{i}}=T_{\mathrm{opt} 1}$ to $T_{1, \mathrm{i}}=2 T_{\mathrm{opt} 1}$. However, when $I_{\mathrm{p}}=-5$, increasing the prestressing level leads to a reduction of the loadcarrying capacity. Moreover, when $T_{1, \mathrm{i}}=2 T_{\mathrm{opt} 1}$, case F3.7 performs best as opposed to case F3.6 at $T_{1, \mathrm{i}}=T_{\mathrm{opt} 1}$, and the ultimate loads of cases F3.6-F3.9 and F3 are numerically close.

\section{3. $I_{\mathrm{p}}=5$}

Figure 22 shows the ultimate loads for cases F3 and F3.6 with varying imperfection amplitudes and pre-cambering index $I_{\mathrm{p}}=5$. For both cases F3 and F3.6, their ultimate loads with the upward imperfection are higher than those with the downward imperfection. For case F3.6, compared to the results with $I_{\mathrm{p}}=-5$ and $I_{\mathrm{p}}=0$ in Figures 12 and 17, its load-carrying capacity with $I_{\mathrm{p}}=5$ is more sensitive in particular to upward imperfections. The reason for this phenomenon appears to be owing to the displacement from pre-cambering also being upward, i.e. in the same direction as the imperfection.

Figure 23 shows the ultimate loads for the different imperfection combinations with $I_{\mathrm{p}}=5$ and a variation of the parameters $\phi_{\mathrm{s}}, a_{1}$ and $\alpha$ at $T_{1, \mathrm{i}}=T_{\mathrm{opt} 1}$. Comparing Figures 23 with 18 , for the doubly-symmetric cases, the load-carrying capacity of these systems with $I_{\mathrm{p}}=5$ is smaller than that with $I_{\mathrm{p}}=0$, but the trends are essentially the same as those with $I_{\mathrm{p}}=0$ and $I_{\mathrm{p}}=-5$. For the mono-symmetric cases, with increasing $\alpha$, the ultimate loads decrease, and case F3.5 performs significantly better than F3.6-F3.9 and F3. Moreover, there is no interactive buckling dominance observed. 


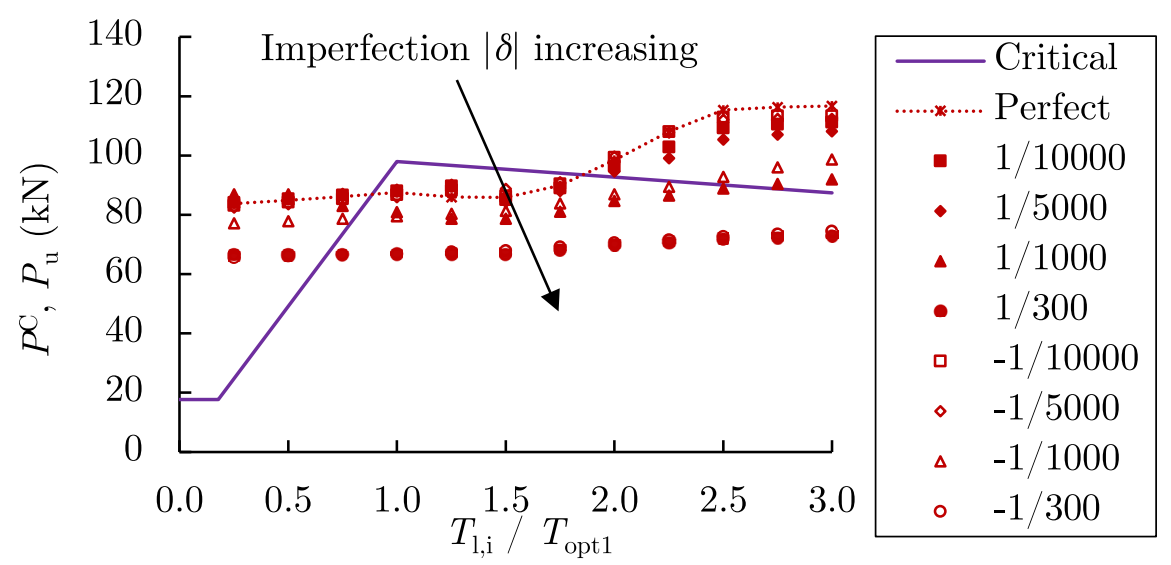

(a) F3

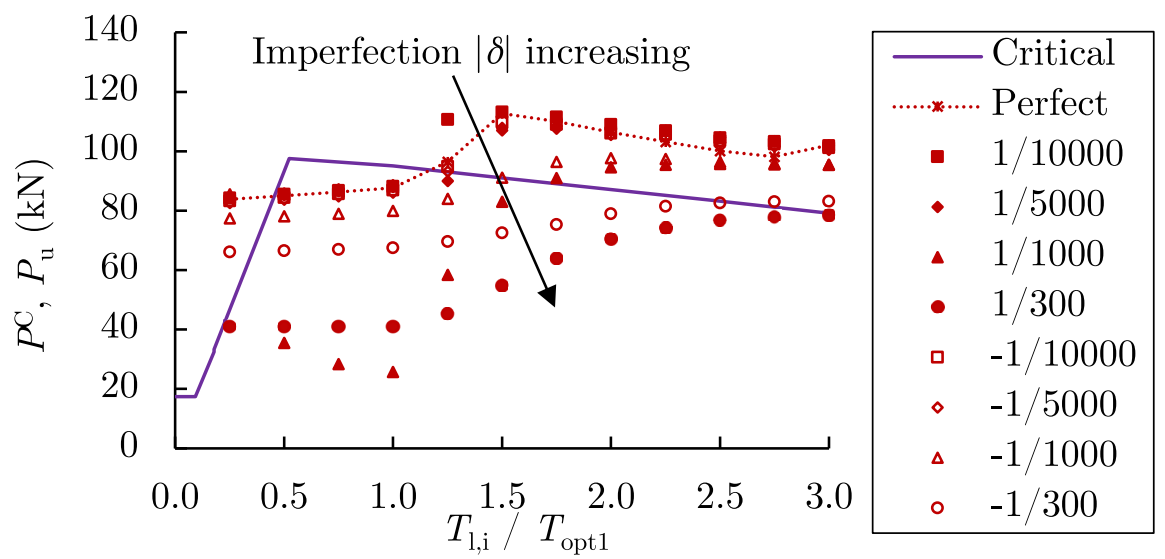

(b) F3.6

Figure 22: Critical buckling (solid line) and ultimate loads (all symbols) for cases F3 and F3.6 with $I_{\mathrm{p}}=5$ and varying $\delta$. Axes and labels are the same as Figure 12. 


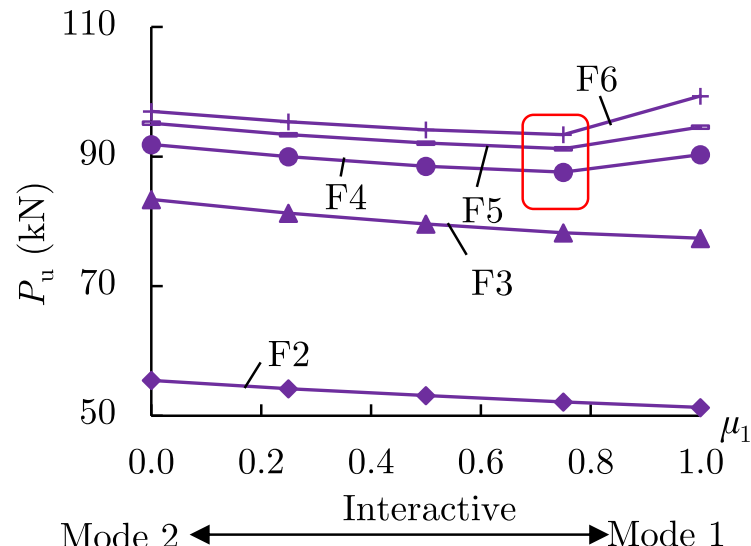

(a) F2-F6

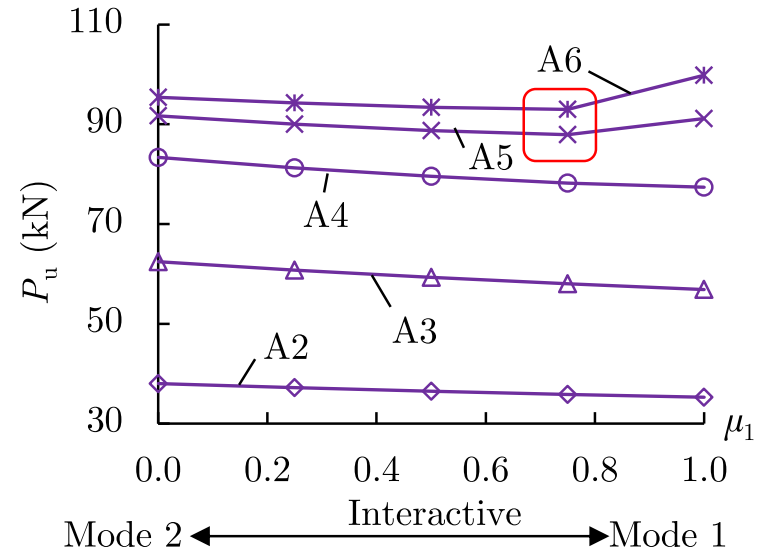

(b) A2-A6

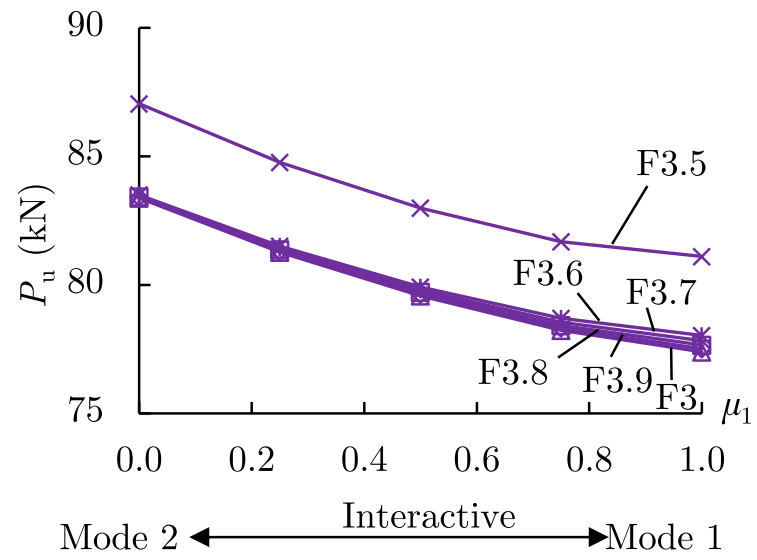

(c) F3.5-F3.9 and F3

Figure 23: Sensitivity to the imperfection combinations represented by $P_{\mathrm{u}}$ versus $\mu_{1}$ with $I_{\mathrm{p}}=5$ and (a) varying $\phi_{\mathrm{s}}$, (b) varying $a_{1}$ and (c) varying $\alpha$. 
The ultimate loads for stayed beam-columns at $T_{1, \mathrm{i}}=T_{\mathrm{opt} 1}$ and $T_{1, \mathrm{i}}=2 T_{\mathrm{opt} 1}$ with $I_{\mathrm{p}}=5$ and varying values of $\phi_{\mathrm{s}}, a_{\mathrm{l}}$ and $\alpha$ are shown in Figures 24, 25, and 26. Compared with

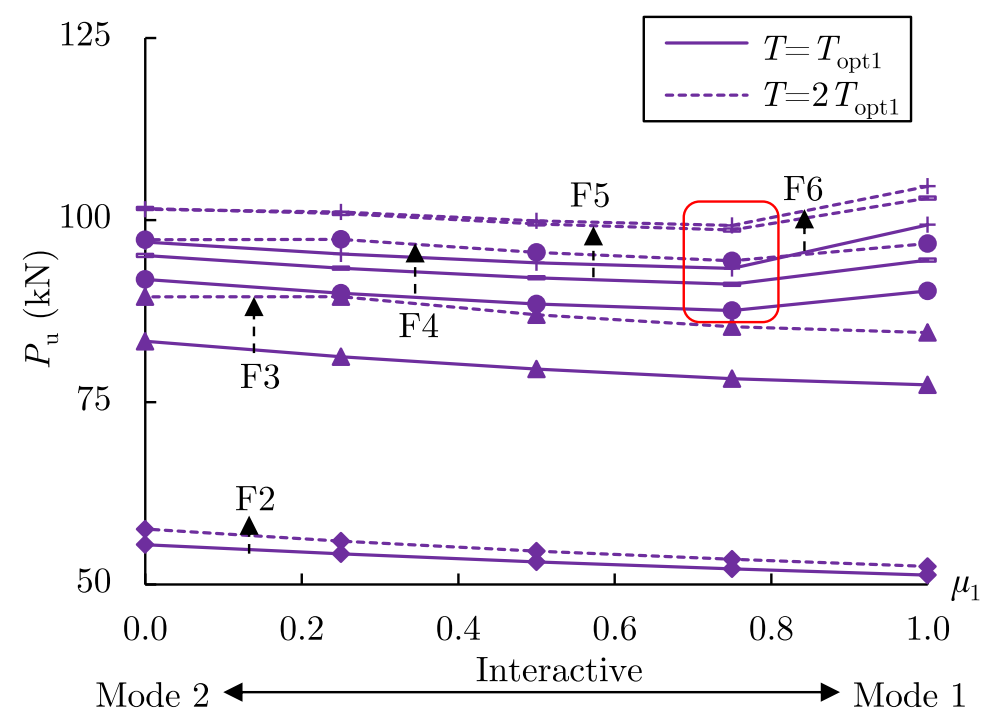

Figure 24: Sensitivity to the downward imperfection combinations represented by $P_{\mathrm{u}}$ versus $\mu_{1}$ with $I_{\mathrm{p}}=5$ and varying $\phi_{\mathrm{s}}$ at $T_{1, \mathrm{i}}=T_{\mathrm{opt} 1}$ and $T_{1, \mathrm{i}}=2 T_{\mathrm{opt} 1}$. Axes and labels are the same as Figure 14 .

the results from these systems with $I_{\mathrm{p}}=-5$ and $I_{\mathrm{p}}=0$, for the doubly-symmetric cases, similar to the results at $T_{1, \mathrm{i}}=T_{\mathrm{opt} 1}$, the load-carrying capacity of these systems with $I_{\mathrm{p}}=5$ is smaller than that with $I_{\mathrm{p}}=0$, but the pattern is essentially the same as that with both $I_{\mathrm{p}}=0$ and $I_{\mathrm{p}}=-5$. Comparing Figure 26 with Figure 21, for the mono-symmetric cases where $I_{\mathrm{p}}=5$, increasing the prestressing level reduces the load-carrying capacity of case F3.5, but for $I_{\mathrm{p}}=0$, this reduction is seen in not only case F3.5 but also case F3.6; for $I_{\mathrm{p}}=-5$, cases F3.5-F3.7 show this trend. Moreover, at $T_{1, \mathrm{i}}=2 T_{\mathrm{opt} 1}$, for cases F3.6 and F3.7, interactive buckling becomes dominant, and the load-carrying capacity of case F3.7 is found to be the highest.

\subsection{Summary}

For the doubly-symmetric cases, the ultimate load, buckling mode and interactive buckling dominance are not sensitive to varying pre-cambering levels, although the load-carrying capacity decreases slightly with changing the pre-cambering level $I_{\mathrm{p}}$ from -5 to 5 . The ultimate loads of the doubly-symmetric cases can be increased by increasing the prestressing levels. However, for the mono-symmetric cases, the buckling and post-buckling behaviour are very sensitive to the level of pre-cambering. For $I_{\mathrm{p}}=-5$ and $I_{\mathrm{p}}=0$, the cases with intermediate crossarm length ratios (i.e. cases F3.6 and F3.7) have relatively high loadcarrying capacities, but the cases with either small or large crossarm length ratios (i.e. cases F3.5 or F3 respectively) have relatively low load-carrying capacities, which implies that the intermediate crossarm length ratios are more suitable for practice; the lowest ultimate loads usually occur in the systems with combined imperfections (i.e. imperfection combinations 2-4 defined in Table 4), which demonstrates the dominance of interactive buckling. 


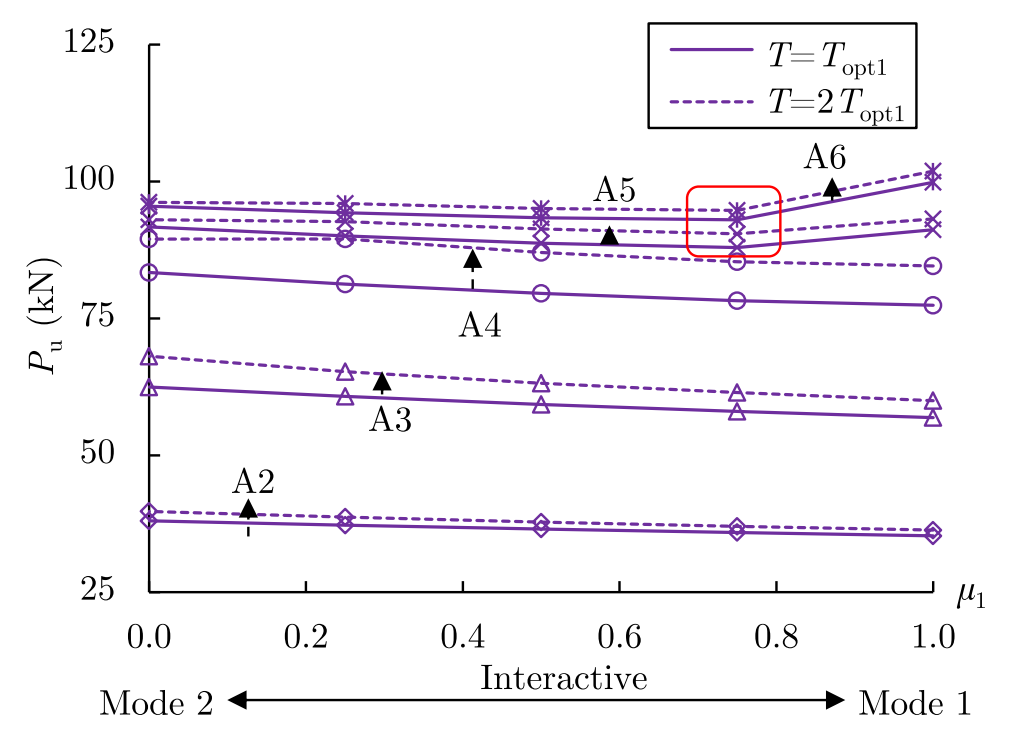

Figure 25: Sensitivity to the downward imperfection combinations represented by $P_{\mathrm{u}}$ versus $\mu_{1}$ with $I_{\mathrm{p}}=5$ and varying $a_{1}$ at $T_{1, \mathrm{i}}=T_{\mathrm{opt} 1}$ and $T_{1, \mathrm{i}}=2 T_{\mathrm{opt} 1}$. Axes and labels are the same as Figure 14 .

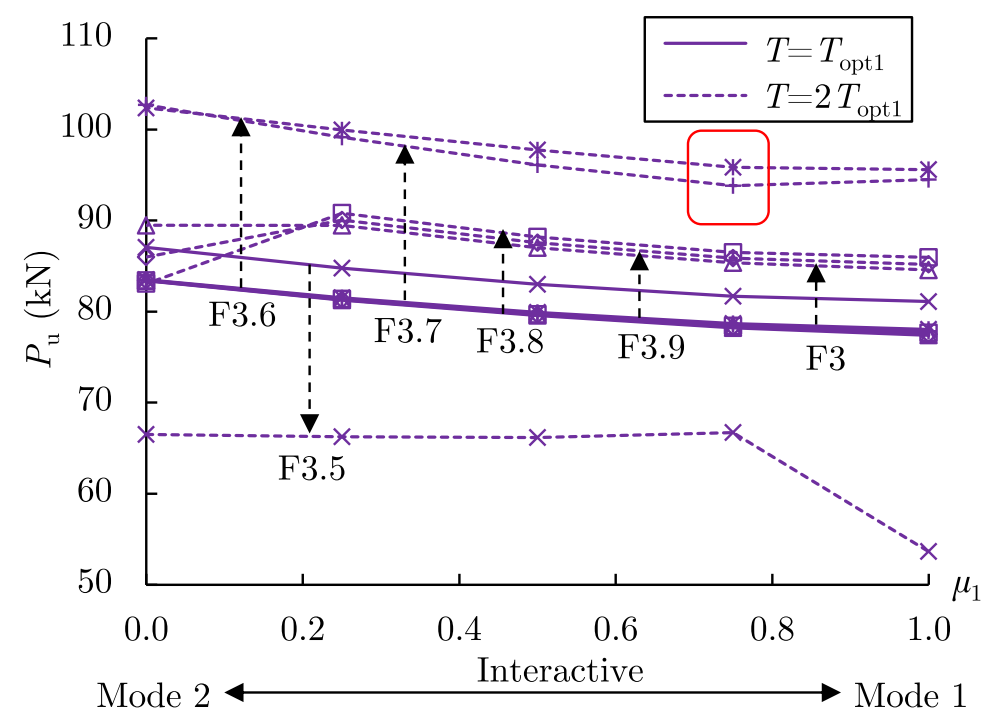

Figure 26: Sensitivity to the downward imperfection combinations represented by $P_{\mathrm{u}}$ versus $\mu_{1}$ with $I_{\mathrm{p}}=5$ and varying $\alpha$ at $T_{1, \mathrm{i}}=T_{\mathrm{opt} 1}$ and $T_{1, \mathrm{i}}=2 T_{\mathrm{opt} 1}$. Axes and labels are the same as Figure 14 . 
For $I_{\mathrm{p}}=5$, reducing the crossarm length ratio increases the load-carrying capacity and interactive buckling does not dominate.

\section{True optimum prestressing force}

In the current section, the true optimum prestressing force is investigated based on the preceding studies on imperfection and prestressing sensitivities. The imperfection amplitude ratio $\delta=1 / 1000$ is adopted, and the imperfection combinations with both the upward and downward principal deflections are taken into account, where the combination leading to the lowest buckling load is adopted in the FE models as shown in Table 5. In terms of the doubly-symmetric systems, for cases F2, A2 and A3, combination 1 is

Table 5: Imperfection combinations and directions for the study of true optimum prestressing forces. Based on the definitions in Table 4, 'U1' and 'D1' represent the upward and downward imperfection with combination 1 respectively, and 'U3' and 'D3' represent the upward and downward imperfection with combination 3 respectively.

\begin{tabular}{cccccc}
\hline Case & F2 & F3 & F4 & F5 & F6 \\
\hline Downward & D1 & D3 & D3 & D3 & D3 \\
Upward & U1 & U3 & U3 & U3 & U3 \\
\hline Case & A2 & A3 & A4 & A5 & A6 \\
\hline Downward & D1 & D1 & D3 & D3 & D3 \\
Upward & U1 & U1 & U3 & U3 & U3 \\
\hline Case & F3.5 & F3.6 & F3.7 & F3.8 & F3.9 \\
\hline Downward & D3 & D3 & D3 & D3 & D3 \\
Upward & U1 & U1 & U1 & U1 & U1 \\
\hline
\end{tabular}

adopted owing to dominant Mode 1 buckling; for cases F3-F6 and A4-A6, combination 3 is adopted as the representative shape to trigger interactive buckling which generally leads to the lowest ultimate loads. In terms of the mono-symmetric cases F3.5-F3.9, combination 1 from Table 4 is adopted for the upward imperfection, since when $\alpha<1$, Mode 1 buckling with an upward deflection leads to a relatively lower load-carrying capacity from the mode interaction study in [33]; combination 3 is adopted as the representative shape to trigger interactive buckling which generally leads to the lowest ultimate loads in downward buckling modes based on the findings from the imperfection sensitivity studies in the present paper and from [33].

To maximize the ultimate load, it has been demonstrated that increasing the initial prestressing level beyond the linearly obtained optimum in [30] is a viable strategy. Apart from determining the true optimum prestressing force based on the prestressing force leading to the maximum ultimate load, structural efficiency is also taken into account, since high prestressing levels usually increase the load-carrying capacity but may reduce the structural efficiency [36], particularly when larger cables are required to introduce higher prestressing forces. In [36], the true optimum prestressing forces for imperfect single-bay 
stayed columns were presented. In order to evaluate the efficiency of the stayed column, two indicators, $\eta$ and $\eta_{\mathrm{s}}$ were proposed, thus:

$$
\eta=\frac{P_{\mathrm{u}}}{A_{\mathrm{c}} f_{\mathrm{y}, \mathrm{req}}}, \quad \eta_{\mathrm{s}}=\frac{P_{\mathrm{u}}}{A_{\mathrm{s}} f_{\mathrm{s}, \text { req }}} \frac{L / 2}{l_{\mathrm{s}}},
$$

where $P_{\mathrm{u}}$ is the ultimate load, while $f_{\mathrm{y}, \text { req }}$ and $f_{\mathrm{s}, \text { req }}$ are the required strengths when $P=P_{\mathrm{u}}$ for the main column and stays respectively; $A_{\mathrm{c}}$ and $A_{\mathrm{s}}$ are the cross-sectional areas of the main column and stays respectively; $L$ and $l_{\mathrm{s}}$ are the lengths of the main column and stays respectively. These two indicators not only consider the load-carrying capacity $P_{\mathrm{u}}$, but also take the material strength of the main column and stays into account. Higher values of these indicators $\eta$ and $\eta_{\mathrm{s}}$ imply that the constituent materials are being utilized to a fuller extent and hence represent more efficient prestressing levels and configurations. For the specific case when $\eta=1.0$, elastic buckling analysis is sufficient and it is unnecessary to consider the resistance of cross-sections. The true optimum prestressing level can be determined by comparing the values of $\eta$ and $\eta_{\mathrm{s}}$, which provides suggestions for optimum structural configurations and prestressing levels. Since the stay lengths and prestressing forces are not equal in mono-symmetric stayed beam-columns [30], the structural efficiency indicator for the stays $\eta_{\mathrm{s}}$ is adjusted slightly with a new definition, thus:

$$
\eta_{\mathrm{s}}=\min \left(\eta_{\mathrm{s}, \mathrm{u} 1}, \eta_{\mathrm{s}, \mathrm{u} 2}, \eta_{\mathrm{s}, 11} \eta_{\mathrm{s}, 12}\right)
$$

where:

$$
\eta_{\mathrm{s}, i}=\frac{P_{\mathrm{u}}}{A_{\mathrm{s}} f_{\mathrm{s}, \mathrm{req}, i}} \frac{L / 2}{l_{i}},
$$

and $i=\{\mathrm{u} 1, \mathrm{u} 2,11,12\}$ corresponding to each stay, as illustrated in Figure 3. However, the definition of the structural efficiency indicator for the main member $\eta$ is unchanged from the doubly-symmetric stayed beam-column definition.

\section{1. $I_{\mathrm{p}}=-5$}

Figures 27, 28 and and 29 show $P_{\mathrm{u}}, \eta$ and $\eta_{\mathrm{s}}$ for varying prestressing levels with the downward pre-cambering level $I_{\mathrm{p}}=-5$ for cases F2-F6, A2-A6, and F3.5-F3.9 and F3 respectively. It can be seen that many high values of the efficiency indicators occur when $T_{1, \mathrm{i}} / T_{\text {opt } 1} \leqslant 0.5$, but these prestressing levels are not recommended owing to the intrinsically low load-carrying capacity at such prestressing levels, which provides requirements of very small column and stay sizes. From Figures 27 and 28, for the doubly-symmetric cases at $T_{1, \mathrm{i}} / T_{\mathrm{opt} 1}=3$, the ultimate loads of most cases do not reach the maximum values, although the gradients of their curves of $P_{\mathrm{u}}$ versus $T_{1, \mathrm{i}} / T_{\mathrm{opt} 1}$ approach zero, but if the aforementioned high values of the efficiency indicators within $T_{1, \mathrm{i}} / T_{\mathrm{opt} 1} \leqslant 0.5$ are discarded, the structural efficiency indicators in fact peak within the range $T_{1, \mathrm{i}} / T_{\mathrm{opt} 1}=[0.5,3]$. From Figure 29, in contrast with the results of the doubly-symmetric cases, the ultimate loads of cases F3.5F3.8 reach their maximum values before approaching $T_{1, \mathrm{i}} / T_{\mathrm{opt} 1}=3$. Comparing Figure 29 with Figures 27 and 28, considering both load-carrying capacity and structural efficiency, for $I_{\mathrm{p}}=-5$, most doubly-symmetric cases perform better than the mono-symmetric cases. 


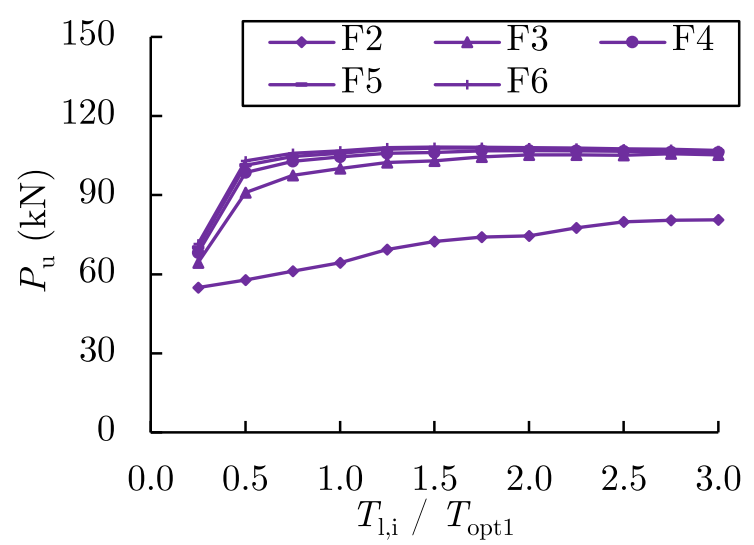

(a) $P_{\mathrm{u}}: \mathrm{U} 1$ or $\mathrm{U} 3$

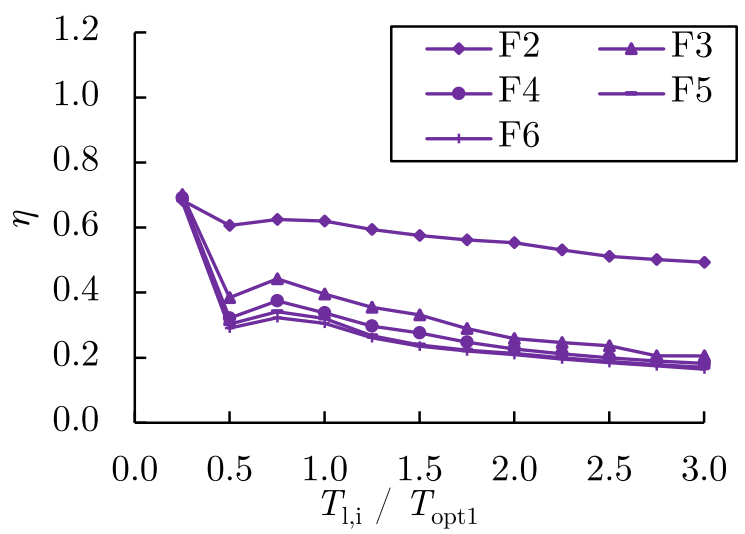

(c) $\eta: \mathrm{U} 1$ or $\mathrm{U} 3$

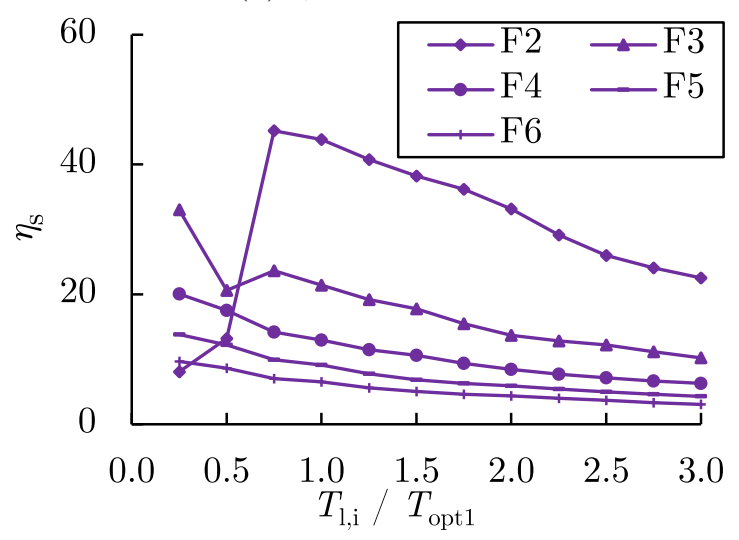

(e) $\eta_{\mathrm{s}}: \mathrm{U} 1$ or $\mathrm{U} 3$

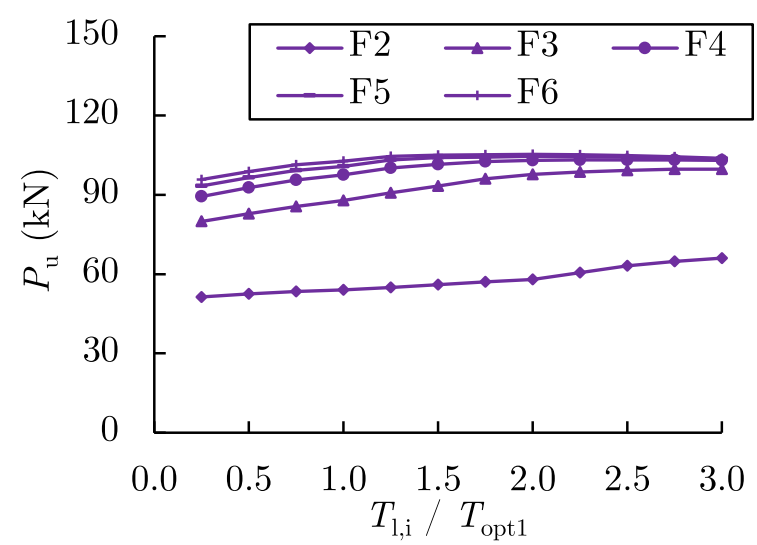

(b) $P_{\mathrm{u}}: \mathrm{D} 1$ or $\mathrm{D} 3$

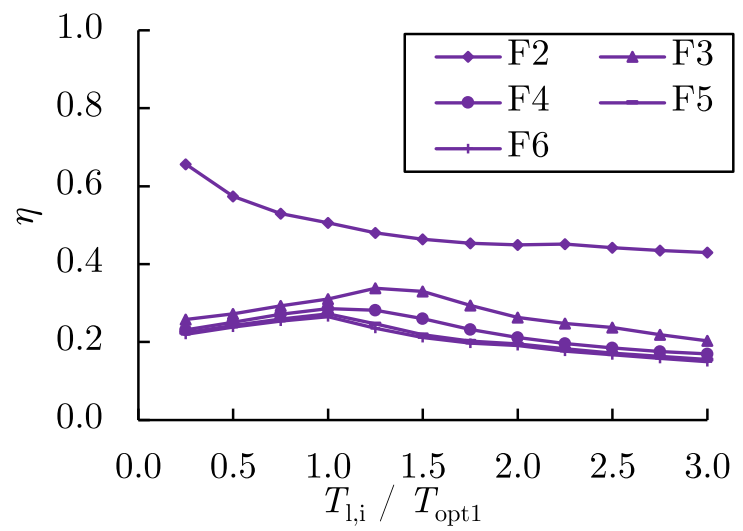

(d) $\eta:$ D1 or D3

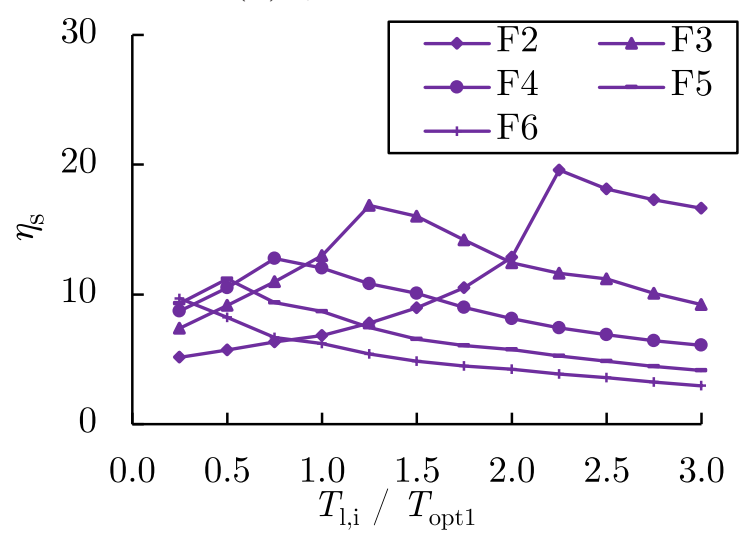

(f) $\eta_{\mathrm{s}}: \mathrm{D} 1$ or D3

Figure 27: True optimum prestressing force for cases F2-F6 with $I_{\mathrm{p}}=-5$. 


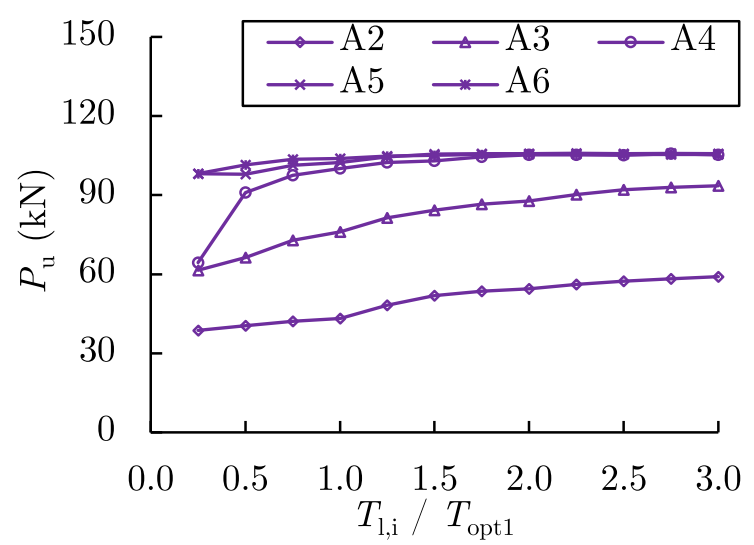

(a) $P_{\mathrm{u}}: \mathrm{U} 1$ or $\mathrm{U} 3$

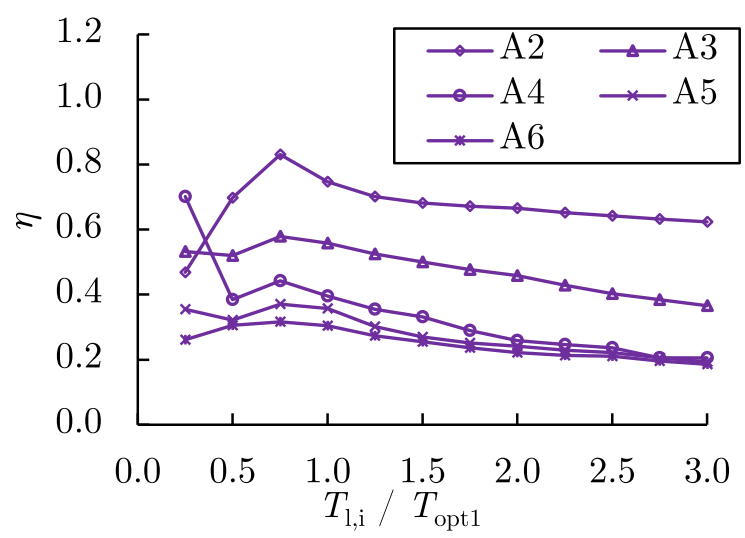

(c) $\eta: \mathrm{U} 1$ or $\mathrm{U} 3$

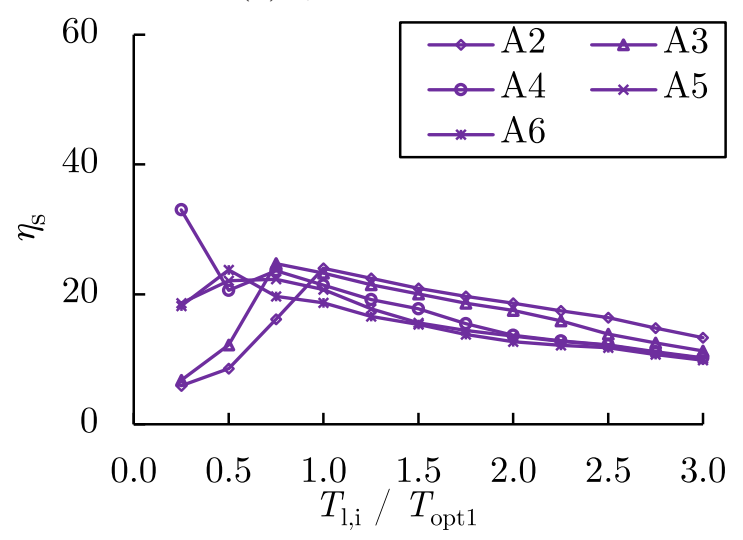

(e) $\eta_{\mathrm{s}}: \mathrm{U} 1$ or $\mathrm{U} 3$

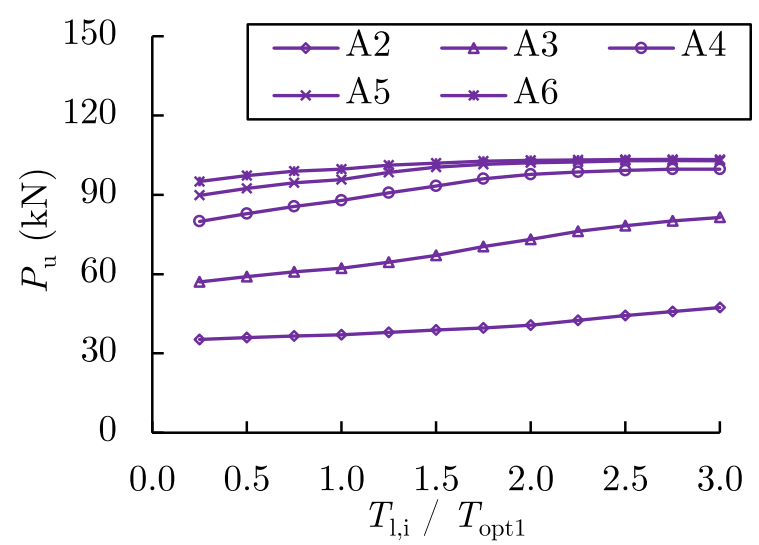

(b) $P_{\mathrm{u}}: \mathrm{D} 1$ or D3

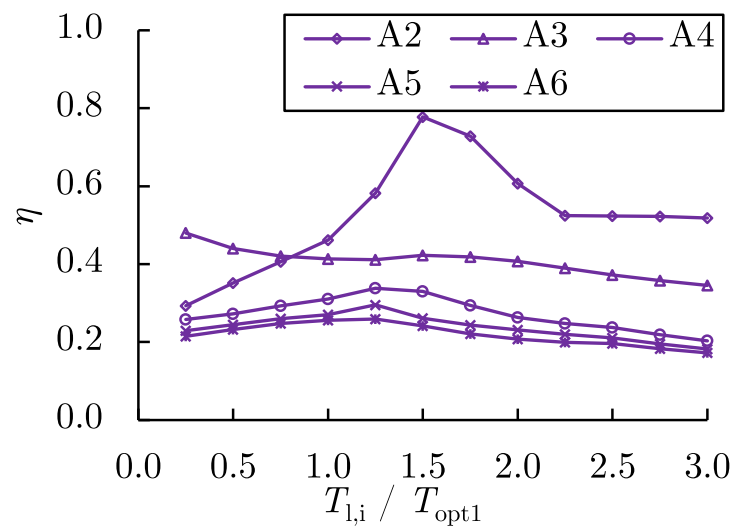

(d) $\eta:$ D1 or D3

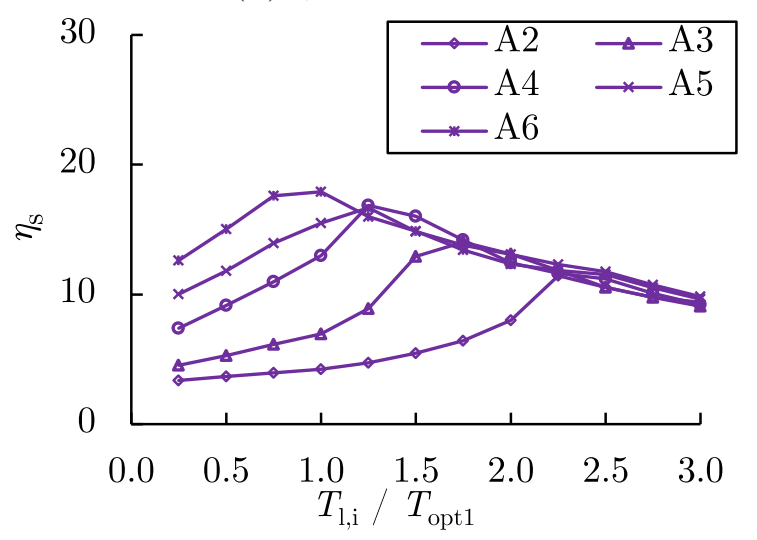

(f) $\eta_{\mathrm{s}}: \mathrm{D} 1$ or D3

Figure 28: True optimum prestressing force for cases A2-A6 with $I_{\mathrm{p}}=-5$. 


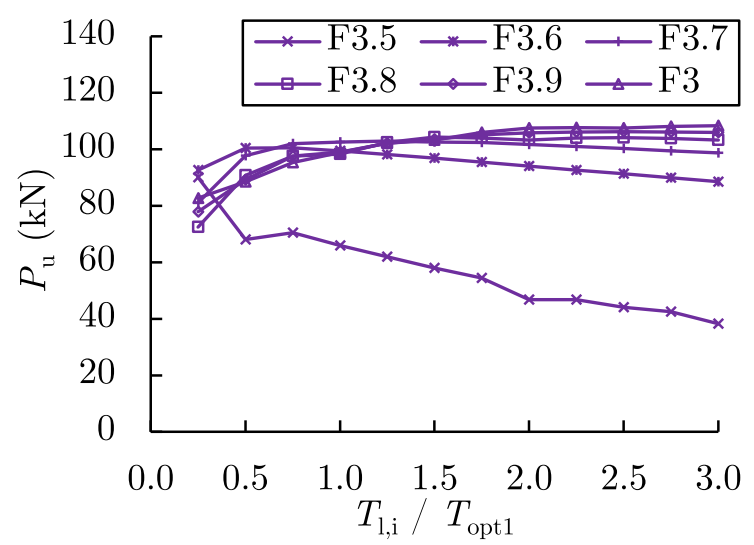

(a) $P_{\mathrm{u}}: \mathrm{U} 1$

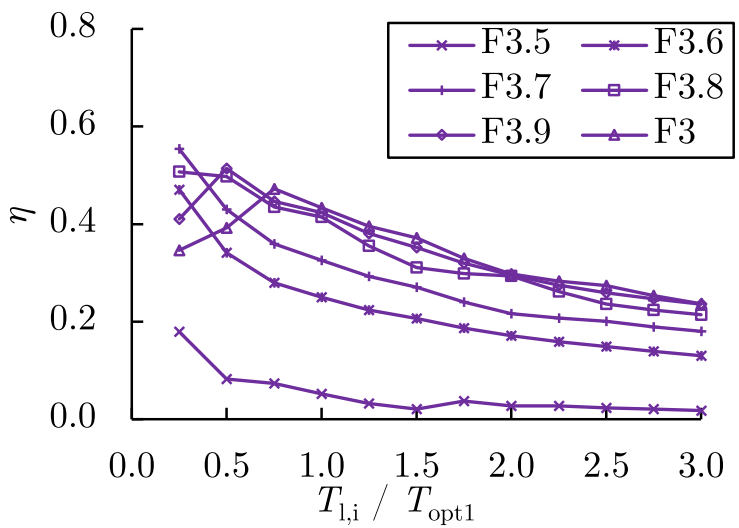

(c) $\eta: \mathrm{U} 1$

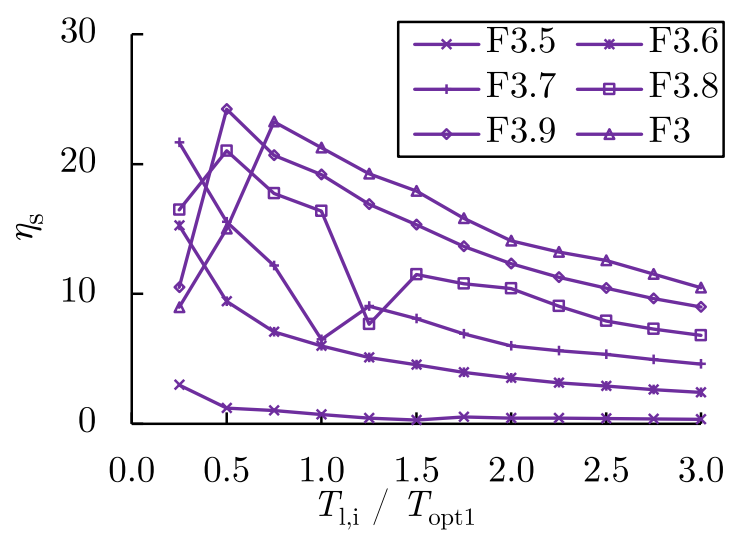

(e) $\eta_{\mathrm{s}}: \mathrm{U} 1$

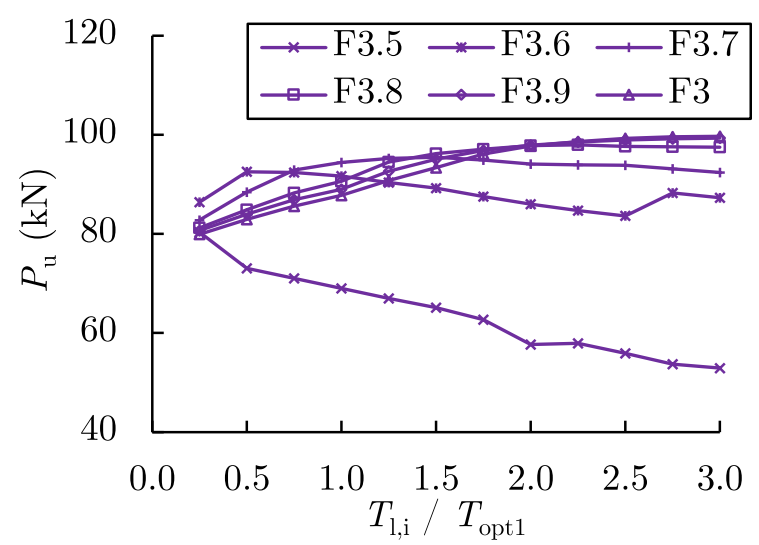

(b) $P_{\mathrm{u}}: \mathrm{D} 3$

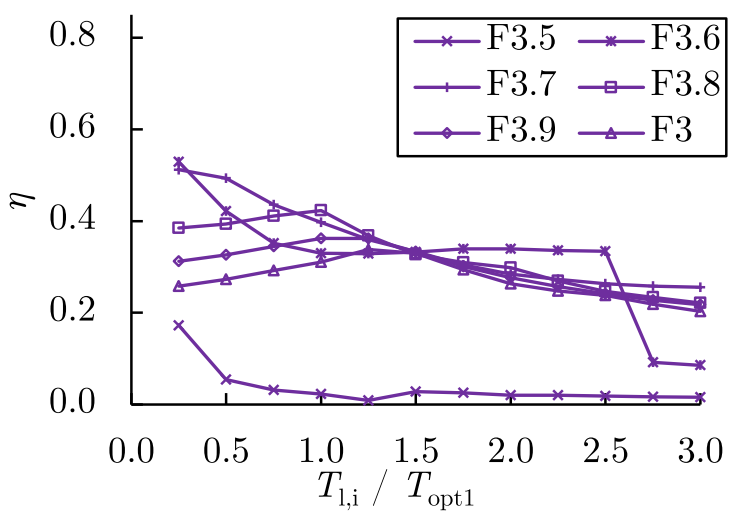

(d) $\eta:$ D3

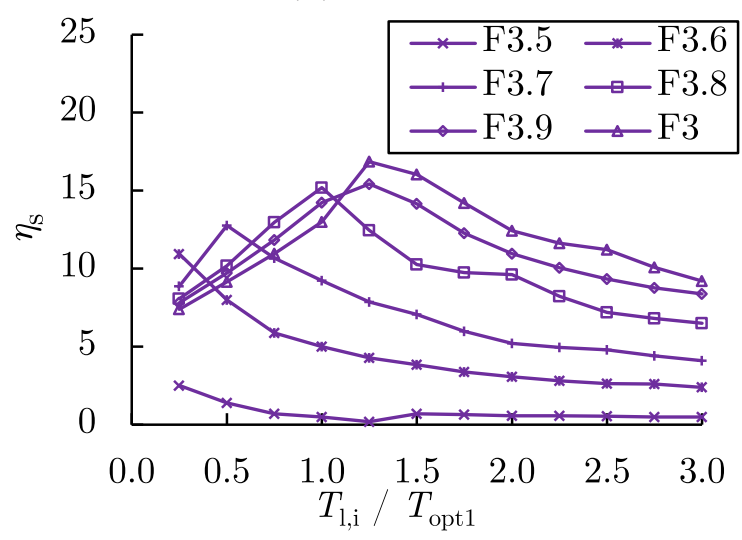

(f) $\eta_{\mathrm{s}}: \mathrm{D} 3$

Figure 29: True optimum prestressing force for cases F3.5-F3.9 and F3 with $I_{\mathrm{p}}=-5$. 


\section{2. $I_{\mathrm{p}}=0$}

Figures 30 and 31 show $P_{\mathrm{u}}, \eta$ and $\eta_{\mathrm{s}}$ for varying prestressing levels with the zero precambering level $I_{\mathrm{p}}=0$ for cases $\mathrm{A} 2-\mathrm{A} 6$, and F3.5-F3.9 and F3 respectively. For cases F2-F6, similar trends with cases A2-A6, presented in Figure 30, are observed. From Figure 30, for the doubly-symmetric cases with upward imperfections, when $T_{1, \mathrm{i}} / T_{\mathrm{opt} 1} \leqslant 1$, the efficiency indicators reduce to a local minimum as $T_{1, \mathrm{i}}$ is increased. Subsequently, this is followed by a local maximum in the efficiency indicators as $T_{1, \mathrm{i}}$ is further increased. From Figure 31, for the mono-symmetric cases with upward imperfections, the extremely high values of efficiency indicators occur when $T_{1, \mathrm{i}} / T_{\mathrm{opt} 1} \leqslant 0.5$ due to the small ultimate loads at such prestressing levels; hence these are again discarded. Comparing Figure 31 with Figure 30, considering both load-carrying capacity and structural efficiency, for the systems with $I_{\mathrm{p}}=0$ and upward imperfections, the doubly-symmetric cases perform better than the mono-symmetric cases, but for the systems with $I_{\mathrm{p}}=0$ and downward imperfections, the reverse is found. For all cases, comparing the results from the systems with $I_{\mathrm{p}}=0$ with those from $I_{\mathrm{p}}=-5$, it is apparent that for $I_{\mathrm{p}}=0$, the required prestressing levels that maximize the values of the structural efficiency indicators are generally higher than that for the case where $I_{\mathrm{p}}=-5$.

\section{3. $I_{\mathrm{p}}=5$}

Figure 32 shows $P_{\mathrm{u}}, \eta$ and $\eta_{\mathrm{s}}$ plotted against varying prestressing levels with upward precambering $I_{\mathrm{p}}=5$ for mono-symmetric cases F3.5-F3.9 and F3. For doubly-symmetric cases F2-F6 and A2-A6, similar trends with cases A2-A6 in Figure 30 are observed, but for $I_{\mathrm{p}}=$ 5 , the reducing section on the curves of ultimate loads versus increasing prestressing levels and the increasing section on the efficiency indicator curves versus prestressing levels when $T_{1, \mathrm{i}} / T_{\mathrm{opt} 1} \leqslant 1$ are more pronounced than when $I_{\mathrm{p}}=0$. Comparing Figure 32 with Figure 31, for the mono-symmetric cases with upward imperfections, for $I_{\mathrm{p}}=5$ the extremely high values of efficiency indicators when $T_{1, \mathrm{i}} / T_{\mathrm{opt} 1} \leqslant 0.5$ are more pronounced than that for $I_{\mathrm{p}}=0$. For $I_{\mathrm{p}}=5$, the mono-symmetric cases perform better than doubly-symmetric cases in terms of both load-carrying capacity and structural efficiency, because the values of the efficiency indicators reach the peak values within $T_{1, \mathrm{i}} / T_{\mathrm{opt} 1}=[1,3]$, and higher structural efficiency can be achieved compared to the doubly-symmetric cases in the same geometric and prestressing arrangements. For all cases, changing $I_{\mathrm{p}}$ from 0 to 5 , the required prestressing levels to maximize the structural efficiency indicators are increased. The reason for this trend is postulated to be that the upward pre-cambering reduces the effect from self-weight and, for $I_{\mathrm{p}}=5$, structural efficiency can become higher with much higher prestressing levels than those for $I_{\mathrm{p}}=0$. Note also that the maximum values of $\eta_{\mathrm{s}}$ are higher when $I_{\mathrm{p}}=5$.

\subsection{Summary}

Although most of the ultimate loads do not reach maximum values within the range $T_{1, \mathrm{i}} / T_{\mathrm{opt} 1}=[1,3]$, the required prestressing forces to maximize structural efficiency are within the range of $T_{1, \mathrm{i}} / T_{\mathrm{opt} 1}=[1,3]$. Within this prestressing range, the maximum values of $\eta$ and $\eta_{\mathrm{s}}$ for each case are shown in Figure 33. In Figures 33(a-b) for doubly-symmetric 


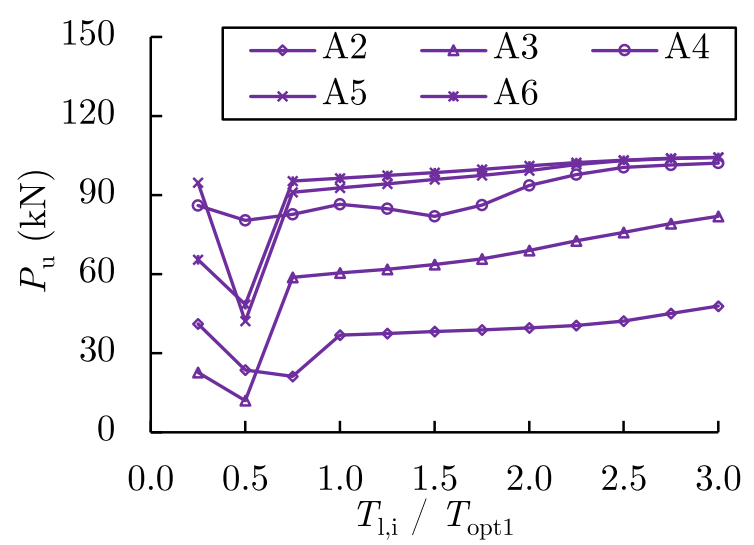

(a) $P_{\mathrm{u}}: \mathrm{U} 1$ or $\mathrm{U} 3$

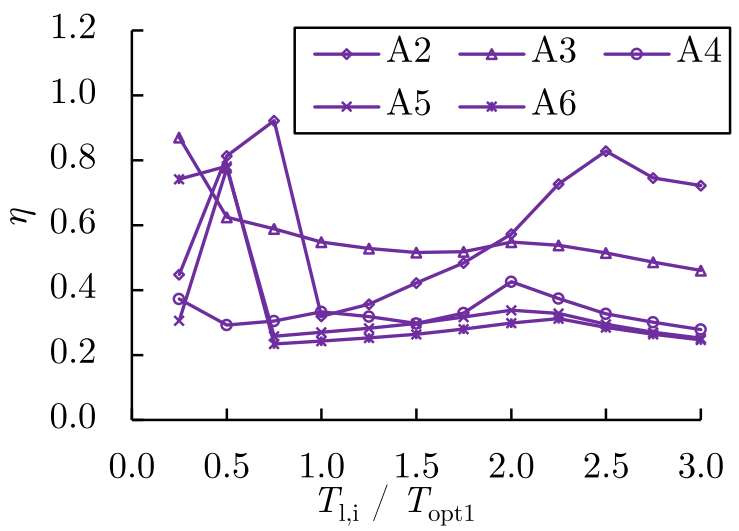

(c) $\eta: \mathrm{U} 1$ or $\mathrm{U} 3$

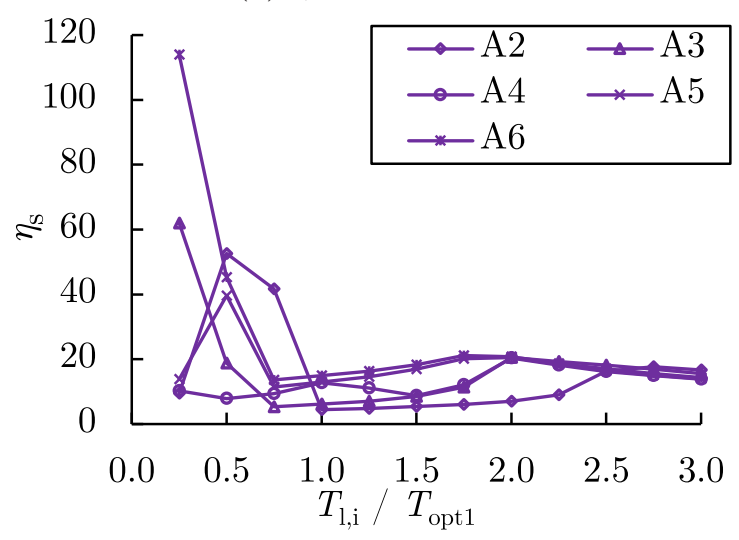

(e) $\eta_{\mathrm{s}}: \mathrm{U} 1$ or $\mathrm{U} 3$

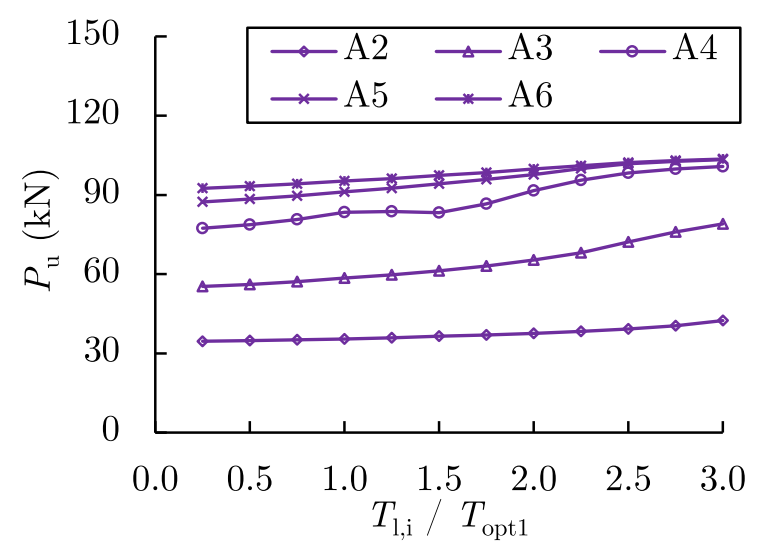

(b) $P_{\mathrm{u}}: \mathrm{D} 1$ or D3

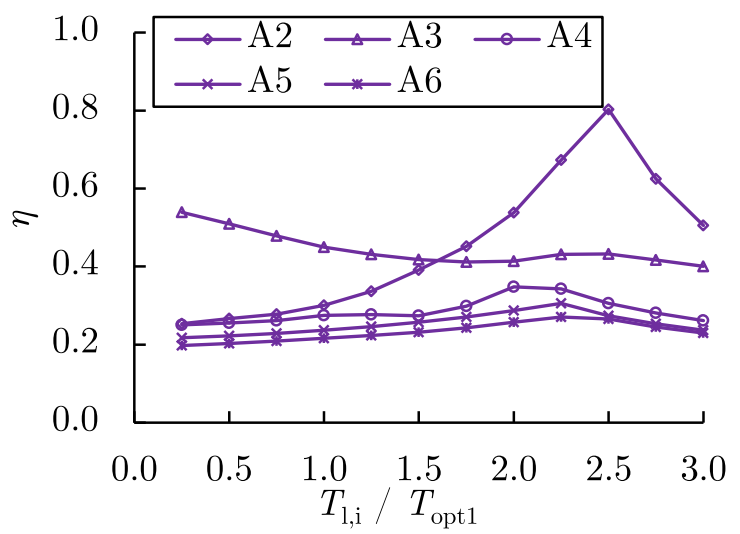

(d) $\eta:$ D1 or D3

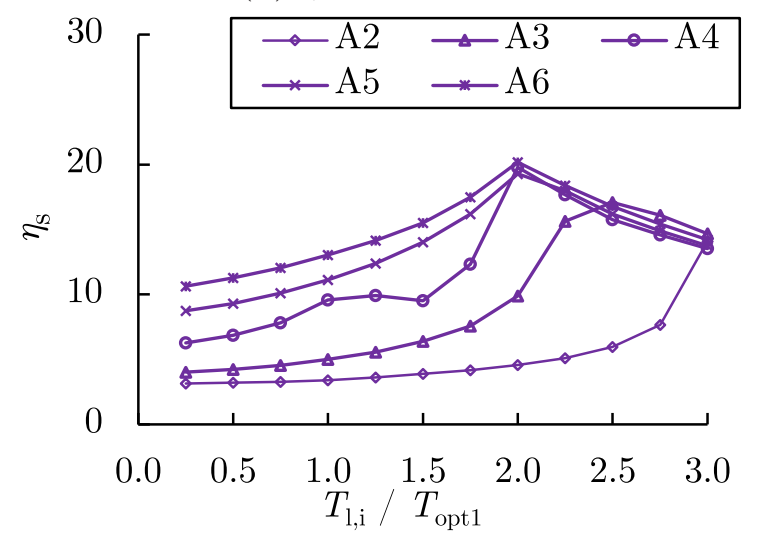

(f) $\eta_{\mathrm{s}}: \mathrm{D} 1$ or D3

Figure 30: True optimum prestressing force for cases A2-A6 with $I_{\mathrm{p}}=0$. 


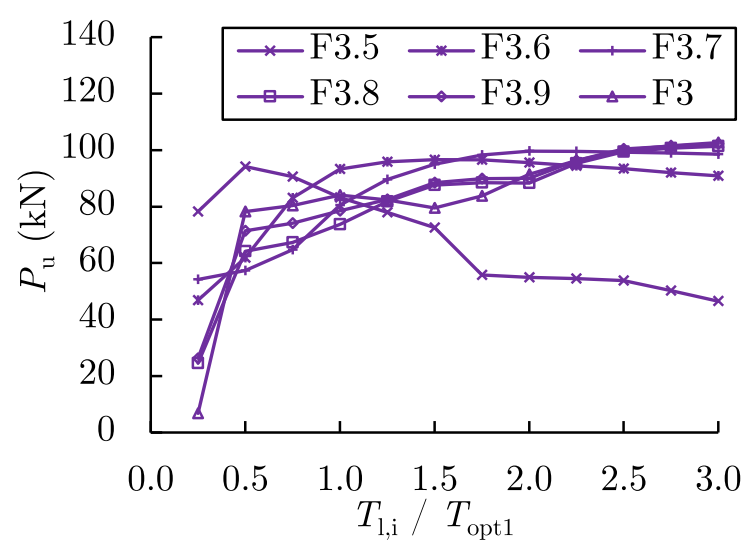

(a) $P_{\mathrm{u}}: \mathrm{U} 1$

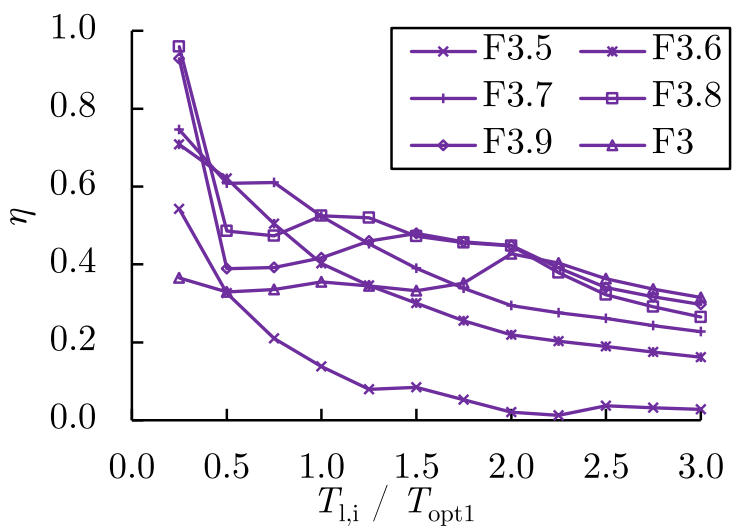

(c) $\eta: \mathrm{U} 1$

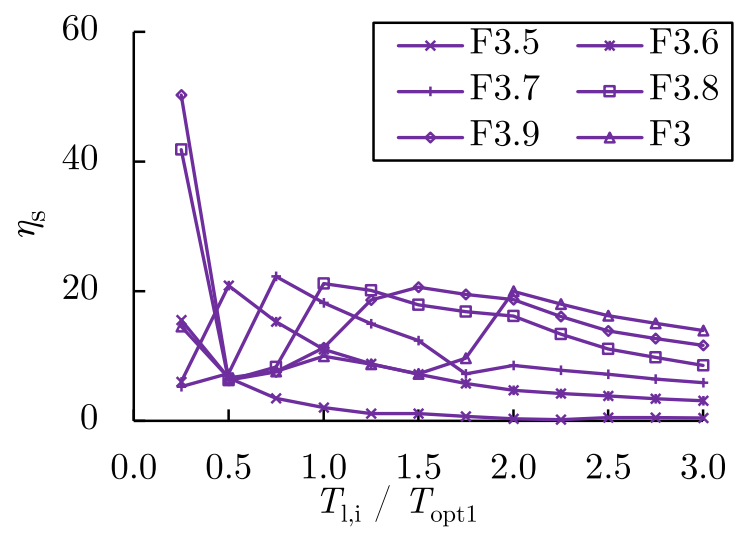

(e) $\eta_{\mathrm{s}}: \mathrm{U} 1$

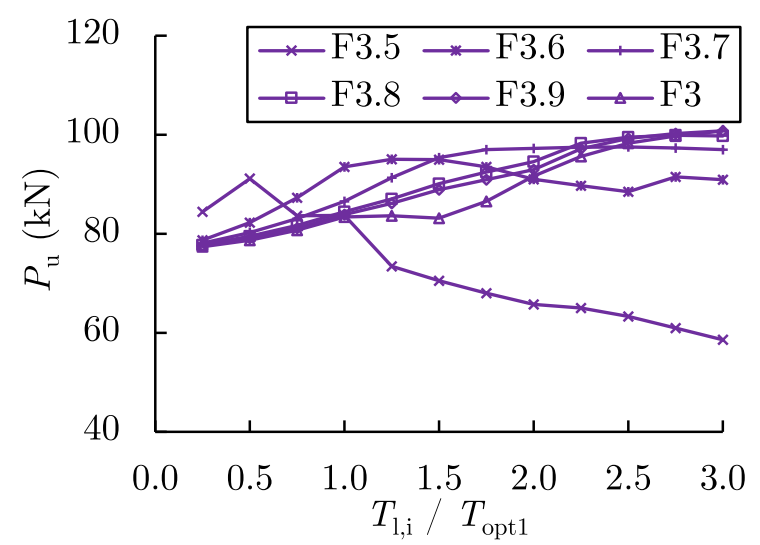

(b) $P_{\mathrm{u}}: \mathrm{D} 3$

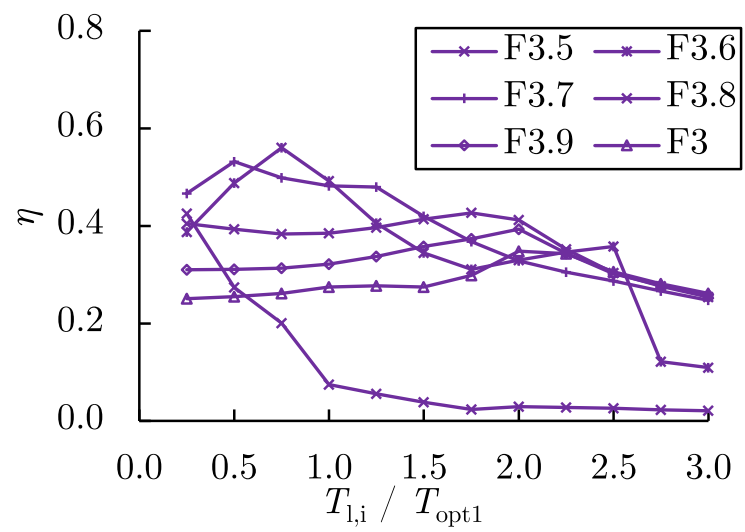

(d) $\eta:$ D3

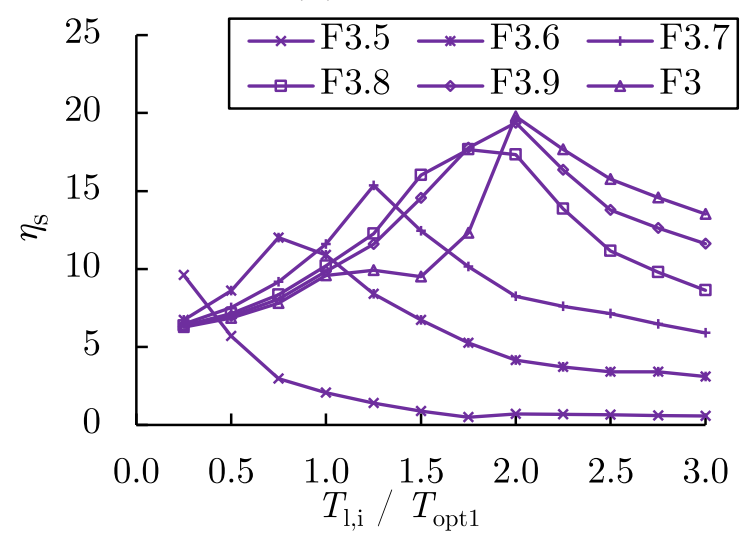

(f) $\eta_{\mathrm{s}}: \mathrm{D} 3$

Figure 31: True optimum prestressing force for cases F3.5-F3.9 and F3 with $I_{\mathrm{p}}=0$. 


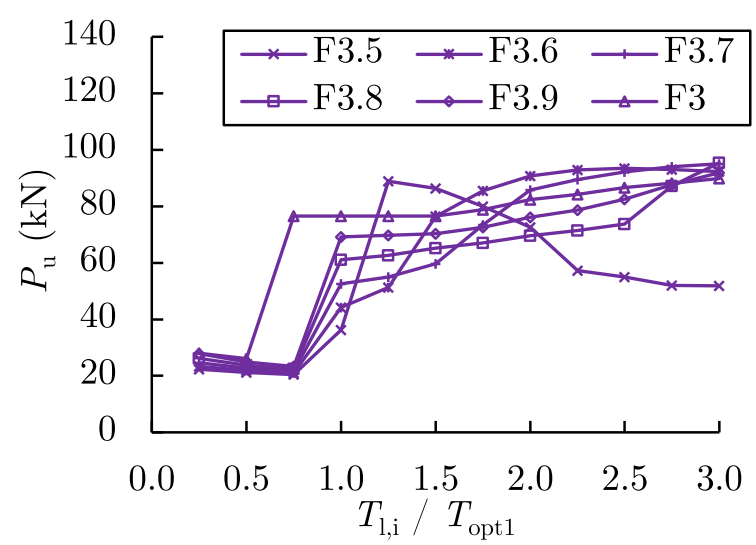

(a) $P_{\mathrm{u}}: \mathrm{U} 1$

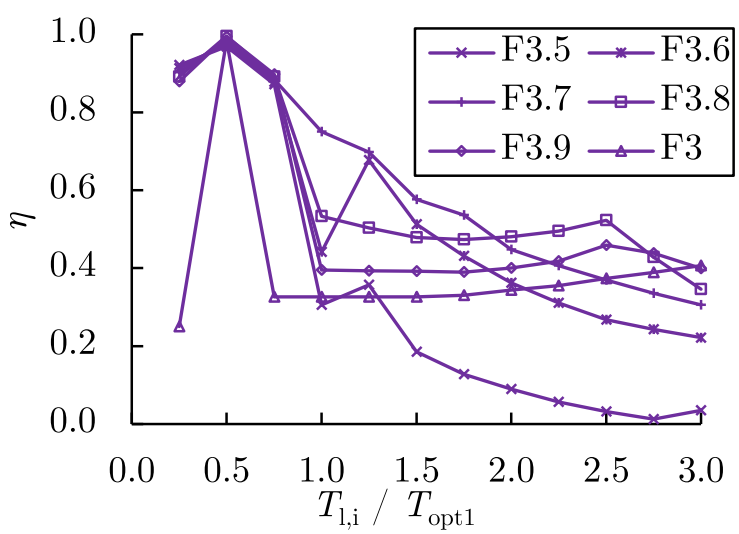

(c) $\eta: \mathrm{U} 1$

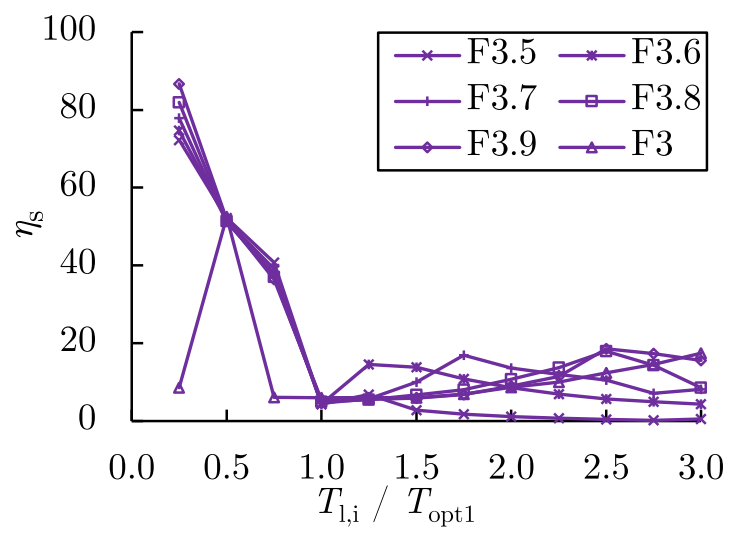

(e) $\eta_{\mathrm{s}}: \mathrm{U} 1$

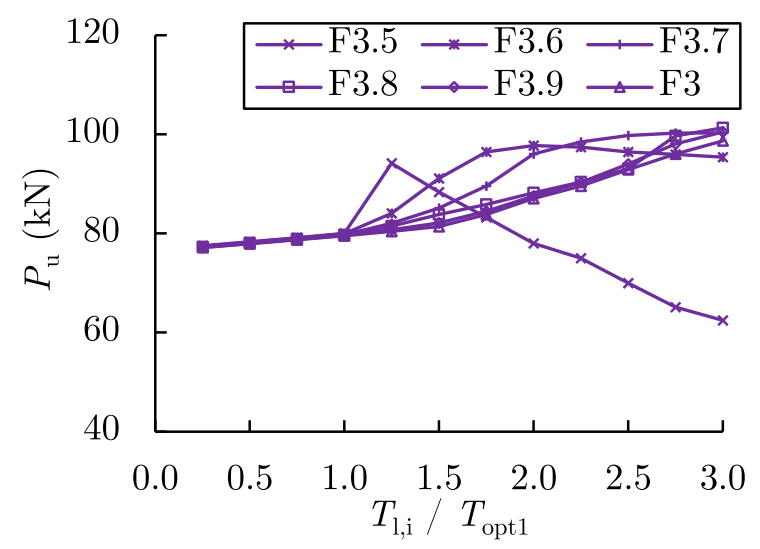

(b) $P_{\mathrm{u}}: \mathrm{D} 3$

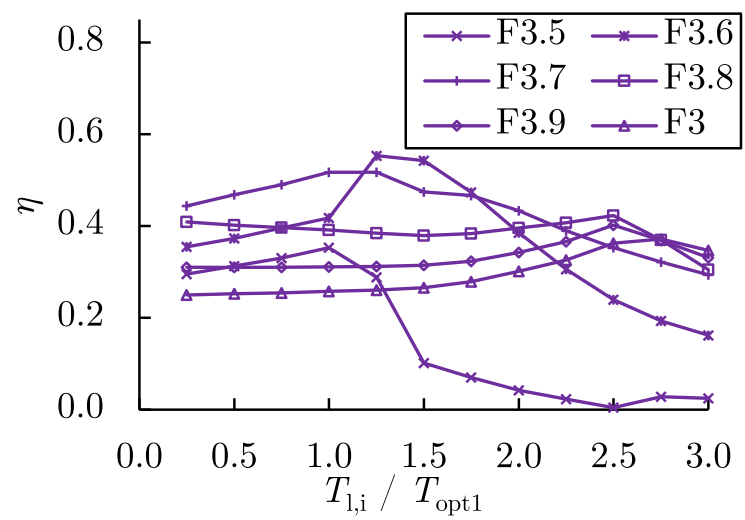

(d) $\eta:$ D3

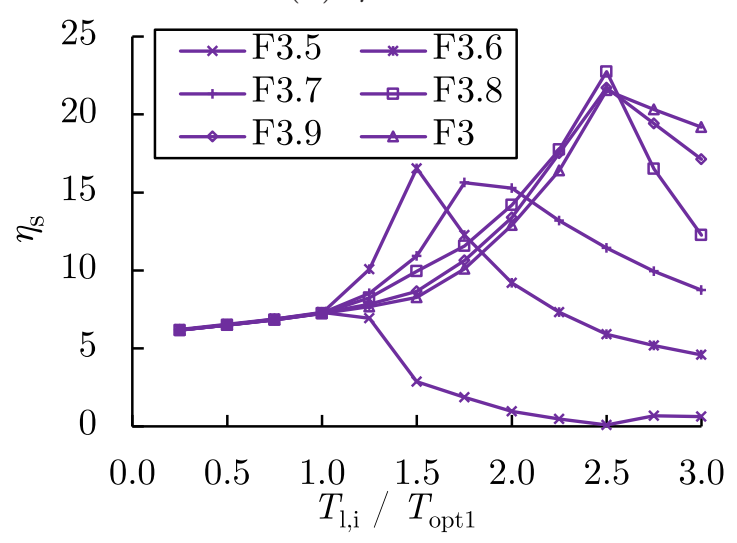

(f) $\eta_{\mathrm{s}}: \mathrm{D} 3$

Figure 32: True optimum prestressing force for cases F3.5-F3.9 and F3 with $I_{\mathrm{p}}=5$. 


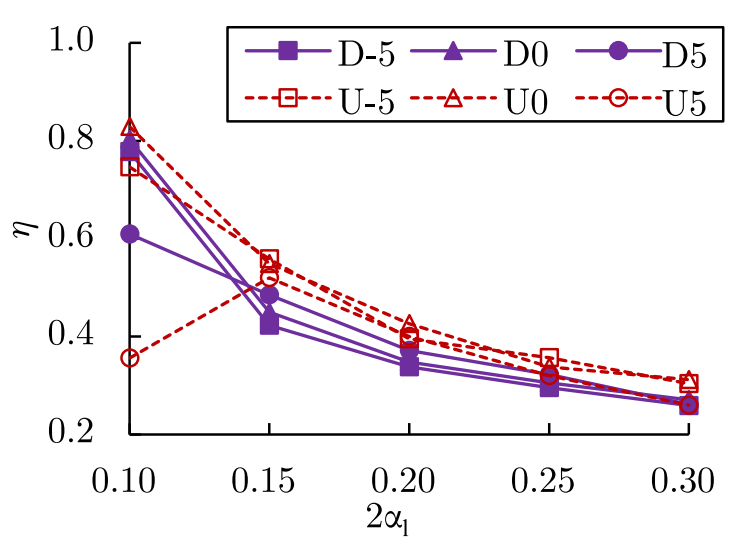

(a) A2-A6: $\eta$

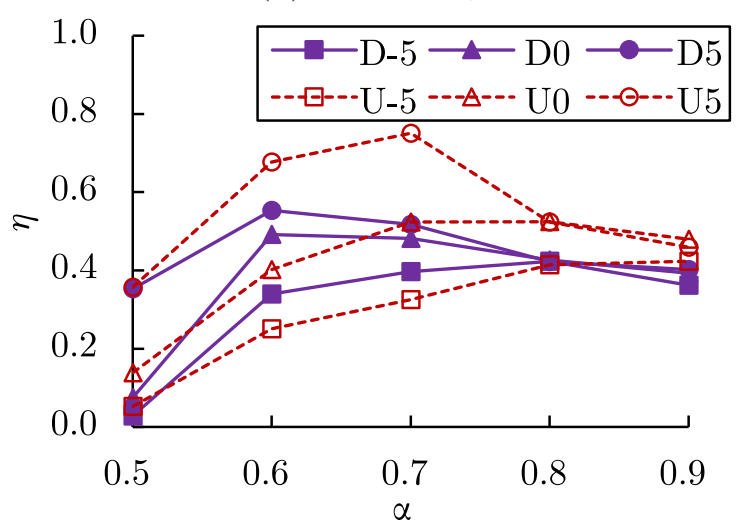

(c) F3.5-F3.9: $\eta$

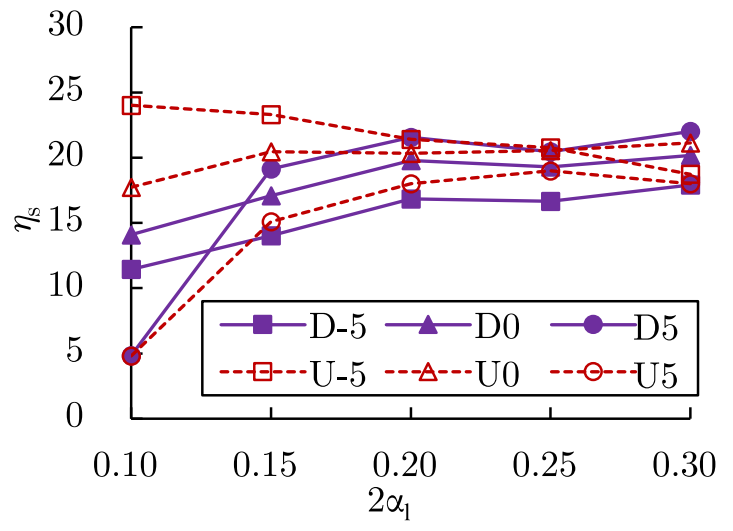

(b) A2-A6: $\eta_{\mathrm{s}}$

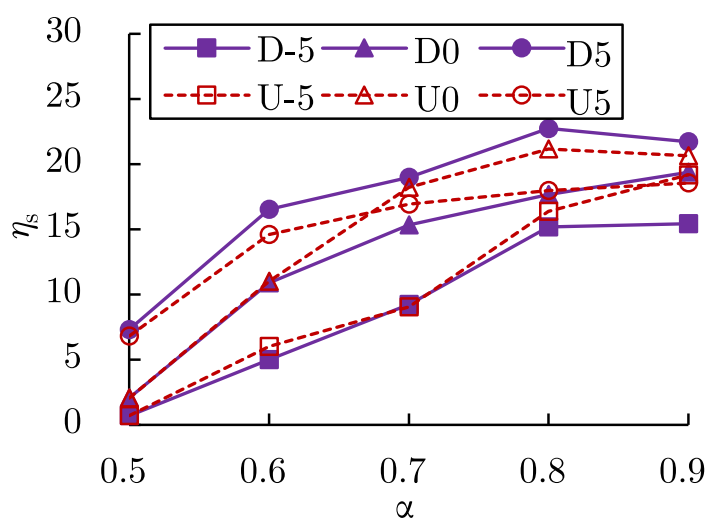

(d) F3.5-F3.9: $\eta_{\mathrm{s}}$

Figure 33: Graphs of $\eta$ and $\eta_{\mathrm{s}}$ for cases A2-A6 and F3.5-F3.9; 'D' and ' $\mathrm{U}$ ' represent downward and upward imperfections respectively; ' -5 ', ' 0 ' and ' 5 ' indicate $I_{\mathrm{p}}=\{-5,0,5\}$ respectively. 
cases, it can be seen that the values of $\eta$ and $\eta_{\mathrm{s}}$ with $I_{\mathrm{p}}=\{-5,5\}$ are sensitive to the imperfection direction. Since the imperfection direction would be random in practice and hence the more severe of the downward (D) and upward (U) imperfections should be considered, for doubly-symmetric cases, zero pre-cambering $\left(I_{\mathrm{p}}=0\right)$ is observed to give better results. In Figures 33(c-d) for mono-symmetric cases, it can be seen that when $I_{\mathrm{p}}=5$, the values of $\eta$ and $\eta_{\mathrm{s}}$ are the greatest in most cases regardless of the imperfection direction. Considering both load-carrying capacity and structural efficiency, the recommended configurations of stayed beam-columns for deliberate pre-cambering levels are presented in Table 6. Moreover, when $T_{1, \mathrm{i}} / T_{\mathrm{opt1}}<1$, although high values of $\eta$ and $\eta_{\mathrm{s}}$ are observed, the

Table 6: Recommended configurations for deliberate pre-cambering levels; ' $D$ ' and ' $U$ ' represent downward and upward imperfections respectively. Ticks $(\checkmark)$ and crosses $(\boldsymbol{x})$ signify configurations that are recommended and not recommended respectively.

\begin{tabular}{cccc}
\hline$I_{\mathrm{p}}$ & -5 & 0 & 5 \\
\hline Mono-symmetric & $\boldsymbol{x}$ & $\checkmark(\mathrm{D})$ & $\checkmark$ \\
Doubly-symmetric & $\checkmark$ & $\checkmark(\mathrm{U})$ & $\boldsymbol{x}$ \\
\hline
\end{tabular}

ultimate loads at such prestressing levels are very small; conversely, when $T_{1, \mathrm{i}} / T_{\mathrm{opt} 1}>3$, although the load-carrying capacity is high, the structural efficiency at such prestressing levels is relatively low. Hence, $T_{1, \mathrm{i}} / T_{\mathrm{opt} 1}<1$ and $T_{1, \mathrm{i}} / T_{\mathrm{opt} 1}>3$ should be avoided for the configurations presented. In addition, $\alpha<0.5$ is not recommended due to the low structural efficiency and issues with highly imperfection sensitive behaviour promoted by strongly unstable post-buckling characteristics.

\section{Concluding remarks}

Based on recent findings that interactive buckling would affect the structural performance of perfect stayed beam-columns under the combined action of axial compression and self-weight acting orthogonally, the structural performance of imperfect stayed beamcolumns has been studied further using FE modelling. Firstly, the relationship between the induced pre-cambering level and the distribution of prestressing between the upper and lower stays were investigated. Then, under varying pre-cambering levels, the ultimate behaviour considering the effects from the varying prestressing levels alongside varying principal imperfection directions, their amplitudes and shapes were investigated. Through applying different imperfection combinations, it transpired that interactive buckling can reduce the ultimate load. Moreover, the true optimum prestressing force for stayed beamcolumns was studied based on the ultimate load and efficiency indicators.

From the study of the prestress and pre-cambering, excessively small crossarm lengths (i.e. $\left.\alpha_{1}<0.05\right)$, stay diameters $\left(\right.$ i.e. $\left.\phi_{\mathrm{s}} /\left(2 r_{\mathrm{c}}\right)<0.14\right)$ and crossarm length ratios (i.e. $\alpha<0.5$ ) should be avoided, since the desired pre-cambering level cannot be achieved by adjusting the prestressing distribution between the upper and lower stays, and the ultimate loads are small in conjunction with highly unstable post-buckling behaviour [30, 33]. The crossarm length ratio $\alpha=0.5$ is not recommended either, but this is primarily owing 
to an intrinsically low structural efficiency. In terms of the buckling and post-buckling behaviour, for doubly-symmetric cases, the structural performance is not particularly sensitive to the pre-cambering level, although the load-carrying capacity decreases slightly by changing the pre-cambering index $I_{\mathrm{p}}$ from -5 to 5 . However, for mono-symmetric cases, interactive buckling dominance, imperfection and prestressing sensitivities, and optimum crossarm length ratios are strongly linked to the pre-cambering levels. In terms of the true optimum prestressing force, although most of the ultimate loads do not reach maximum values within $T_{1, \mathrm{i}} / T_{\mathrm{opt} 1}=[1,3]$, the structural efficiency are indeed maximized within this range. With changing $I_{\mathrm{p}}$ from -5 to 5 , the required prestressing forces to obtain the peak values of the structural efficiency indicators increase. Therefore, $T_{1, \mathrm{i}} / T_{\mathrm{opt} 1}=[1,3]$ is recommended, since this range achieves a good balance of load-carrying capacity and structural efficiency. Moreover, doubly-symmetric stayed beam-columns perform better with zero pre-cambering, while mono-symmetric cases perform better with an upward pre-camber.

Since the performance of the stayed beam-columns under axial compression in conjunction with self-weight has been studied in some depth, the authors are now investigating the combination of axial compression and other bending arrangements. This would enable guidance for designers to be established in future for more general loading cases.

\section{Acknowledgement}

Financial support for the lead author was provided by the China Scholarship Council.

\section{References}

[1] K. H. Chu, S. S. Berge, Analysis and design of struts with tension ties, ASCE Journal of the Structural Division 89 (1963) 127-163.

[2] H. R. Mauch, Optimum design of columns supported by tension ties, ASCE Journal of Structural Engineering 93 (3) (1967) 201-220.

[3] R. J. Smith, J. S. Ellis, G. T. McCaffrey, Buckling of a single-crossarm stayed column, ASCE Journal of the Structural Division 101 (1) (1975) 249-268.

[4] E. Belenya, Prestressed load-bearing metal structures, Moscow: Mir Publishers, 1977.

[5] H. H. Hafez, M. C. Temple, J. S. Ellis, Pretensioning of single-crossarm stayed columns, ASCE Journal of the Structural Division 105 (2) (1979) 359-375.

[6] M. C. Temple, Buckling of stayed columns, ASCE Journal of the Structural Division 103 (4) (1977) 839-851.

[7] M. C. Temple, M. V. Prakash, J. S. Ellis, Failure criteria for stayed columns, ASCE Journal of Structural Engineering 110 (1984) 2677-2689.

[8] K. C. Wong, M. C. Temple, Stayed column with initial imperfection, ASCE Journal of the Structural Division 108 (1982) 1623-1640. 
[9] A. K. Jemah, F. W. Williams, Parametric experiments on stayed columns with slender bipods, International Journal of Mechanical Sciences 32 (1990) 83-100.

[10] R. R. De Araujo, S. A. L. De Andrade, P. C. G. d. S. Vellasco, J. G. S. Da Silva, L. R. O. De Lima, Experimental and numerical assessment of stayed steel columns, Journal of Constructional Steel Research 64 (2008) 1020-1029.

[11] A. I. Osofero, M. A. Wadee, L. Gardner, Experimental study of critical and postbuckling behaviour of prestressed steel stayed columns, Journal of Constructional Steel Research 79 (2012) 226-241.

[12] A. I. Osofero, M. A. Wadee, L. Gardner, Numerical studies on the buckling resistance of prestressed stayed columns, Advances in Structural Engineering 16 (2013) 487-498.

[13] P. Li, M. A. Wadee, J. Yu, N. G. Christie, M. Wu, Stability of prestressed stayed steel columns with a three branch crossarm, Journal of Constructional Steel Research 122 (2016) 274-291.

[14] P. Zhou, Y.-L. Guo, Y.-L. Pi, Numerical investigations of multiple cross-arm pretensioned cable stayed BRBs with pin-ended stays, Journal of Constructional Steel Research 148 (2018) 206-232.

[15] S. L. Chan, G. P. Shu, Z. T. Lü, Stability analysis and parametric study of pre-stressed stayed columns, Engineering Structures 24 (2002) 115-124.

[16] D. Saito, M. A. Wadee, Post-buckling behaviour of prestressed steel stayed columns, Engineering Structures 30 (5) (2008) 1224-1239.

[17] D. Saito, M. A. Wadee, Buckling behaviour of prestressed steel stayed columns with imperfections and stress limitation, Engineering Structures 31 (1) (2009) 1-15.

[18] D. Saito, M. A. Wadee, Numerical studies of interactive buckling in prestressed steel stayed columns, Engineering Structures 31 (2) (2009) 432-443.

[19] M. A. Wadee, L. Gardner, T. A. Hunt, Buckling mode interaction in prestressed stayed columns, Proceedings of the Institution of Civil Engineers - Structures and Buildings 166 (2013) 403-412.

[20] J. Yu, M. A. Wadee, Mode interaction in triple-bay prestressed stayed columns, International Journal of Non-Linear Mechanics 88 (2017) 47-66.

[21] P. Li, X. Liu, C. Zhang, Interactive buckling of cable-stiffened steel columns with pinconnected crossarms, Journal of Constructional Steel Research 146 (2018) 97-108.

[22] M. A. Wadee, L. Gardner, A. I. Osofero, Design of prestressed stayed columns, Journal of Constructional Steel Research 80 (2013) 287-298. 
[23] T. Tankova, L. S. da Silva, J. P. Martins, Stability design of cable-stayed columns: Existing methods and future perspectives, Steel Construction 12 (4) (2019) 309-317.

[24] J. Gosaye, L. Gardner, M. A. Wadee, M. E. Ellen, Tensile performance of prestressed steel elements, Engineering Structures 79 (2014) $234-243$.

[25] J. Gosaye, L. Gardner, M. A. Wadee, M. E. Ellen, Compressive behaviour and design of prestressed steel elements, Structures 5 (2016) 76-87.

[26] N. Hadjipantelis, L. Gardner, M. A. Wadee, Prestressed cold-formed steel beams: Concept and mechanical behaviour, Engineering Structures 172 (2018) 1057 - 1072.

[27] N. Hadjipantelis, L. Gardner, M. A. Wadee, Design of prestressed cold-formed steel beams, Thin-Walled Structures 140 (2019) 565 - 578.

[28] N. Hadjipantelis, L. Gardner, M. A. Wadee, Finite-element modeling of prestressed cold-formed steel beams, Journal of Structural Engineering 145 (10) (2019) 04019100.

[29] M. A. Wadee, N. Hadjipantelis, J. B. Bazzano, L. Gardner, J. A. Lozano-Galant, Stability of steel struts with externally anchored prestressed cables, Journal of Constructional Steel Research 164 (2020) 105790.

[30] K. Wu, M. A. Wadee, L. Gardner, Stability and ultimate behaviour of prestressed stayed beam-columns, Engineering Structures 201 (2019) 109723.

[31] S. Krishnan, Cable-stayed columns and their applications in building structures, Journal of Building Engineering 27 (2020) 100984.

[32] F. Madrazo-Aguirre, M. A. Wadee, A. M. Ruiz-Teran, Non-linear stability of underdeck cable-stayed bridge decks, International Journal of Non-Linear Mechanics 77 (2015) 28-40.

[33] K. Wu, M. A. Wadee, L. Gardner, Interactive buckling in prestressed stayed beamcolumns, International Journal of Mechanical Sciences 174 (2020) 105479.

[34] P. R. Everall, G. W. Hunt, Mode jumping in the buckling of struts and plates: a comparative study, International Journal of Non-Linear Mechanics 35 (2000) 10671079 .

[35] N. S. Trahair, M. A. Bradford, D. A. Nethercot, L. Gardner, The behaviour and design of steel structures to EC3, 4th Edition, Taylor \& Francis, London, 2008.

[36] D. Saito, M. A. Wadee, Optimal prestressing and configuration of stayed columns, Proceedings of the Institution of Civil Engineers - Structures and Buildings 163 (5) (2010) 343-355.

[37] J. Yu, M. A. Wadee, Optimal prestressing of triple-bay prestressed stayed columns, Structures 12 (2017) 132-144. 
[38] E. Riks, An incremental approach to the solution of snapping and buckling problems, International Journal of Solids and Structures 15 (1979) 529-551.

[39] M. A. Wadee, Effects of periodic and localized imperfections on struts on nonlinear foundations and compression sandwich panels, International Journal of Solids and Structures 37 (2000) 1191-1209.

[40] ABAQUS, Version 6.14 Documentaion, Dassault Systèmes Simulia Corp., Providence, RI, USA (2016). 